

\section{TRITA-EPP-77-11 \\ ON THE ROLE OF MAGNETIC \\ MIRRORING IN THE AURORAL PHENOMENA}

Walter Lennartsson

December 1976

Department of Plasma Physics Royal Institute of Technology 10044 STOCKHOLM 70, Sweden 
ON THE ROLE OF MAGNETIC MIRRORING IN THE AURORAL, PHENOMENA Walter Lennartsson

Royal Institute of Technology, Department of Plasma Physics, Stockholm 70, Sweden

Abstract. On the basis of field and particle observations, it is suggested that a bright auroral display is a part of a magnetosphere-ionosphere cument system which is fed by a charge-separation process in the outer magnetosphere (or the solar wind). The upward magnetic-field-aligned current is flowing out of the display, carried mainly by downflowing electrons from the hot-panticle populations in the outer magnetosphere (the ambient cold electrons being depleted at high altitudes). As a result of the magnetic mirroning of these downflowing current carriers, a large potential drop is set up along the magnetic field, increasing both the number flux and the kinetic energy of precipitating electrons. It is found that this simple basic model, when combined with wave-particle interactions, may be able to explain a highly diversified selection of auroral particle observations. It may thus be possible to explain both "inverted- $V$ " events and auroral rays in terms of a static parallel electric field, and the electric field may be compatible with a strongly variable pitch-angle distribution of the precipitating electrons, incluaing distributions peaked at $90^{\circ}$ as well as $0^{\circ}$. This model may also provide a simple explanation of the simultaneous precipitation of electrons and collimated positive ions. 


\section{Introduction}

One of the most puzzIing problems in magnetospheric physics today is how to understand the complex processes that cause energization and precipitation of the auroral particles. A great variety of ideas have been presented in the literature to explain different observed phenomena, but so far very little has been achieved in getting a unified picture. For an illustration of the complexity of auroral particle observations, see, for example, O'Brien and Reasoner (1971).

The purpose of this paper is to emphasize the potential central role of magnetic mirroring in the complex auroral phenomena and to suggest that a proper consideration of the magnetic mirroring may greatly simplify the present picture. The reader who only wants a brief orientation may read Sections 2,15 and 16 .

For reference purposes we need a rough division of current ideas about auroral particle acceleration. The main ideas may be grouped into the following three basic categories:

(a) The precipitating particles have already attained their final energy when leaving the equatorial region of the magnetosphere. It is frequently assumed that the auroral particles get their final energy, by, for instance, betatron and Fermi acceleration during their drift motion into and filling of the plasma sheet reservoir with energetic particles. In order for a sufficient flux of the energized particles to reach down to the atmosphere, despite the strong mirroring effect of the geomagnetic field, a final pitch-angle scattering mechanism (by means of wave-particle interactions) may be needed. See, for instance, Kennel and Petschek (1966). Some measurements of the nearearth plasma sheet seem to indicate a sufficient energy flux of particles with appropriate energies 
for producing even the most intense auroral precipitation (Frank, 1971; Vasyliunas, 1970). For a very brief review of this kind of lange scale energization, see Heikkila (1974).

(b) The precipitating particles in general, or at least some of them, gain additional energy at the expense of a trapped particle component through which they pass on theix way down. Alternatively, this energy transfer may be from one precipitating component to the other. According to this view the energy yielding particles having an unstable velocity distribution produce plasma waves, the energy of which is absorbed by the precipitating particles in an ordered manner. A crucial point is then to have the precipitating particles increase preferentially their downward field-aligned velocity. For examples of these kinds of ideas see Gary et al (1968), Laval and Pellat (1970), Perkins (1968) and Swift (1970).

(c) The precipitating particles fall through an electrostatic potential gradient along the magnetic field lines. This requines, of course, a drastic reduction of the parallel current-carrying capability of the magnetospheric and ionospheric plasmas relative to what has been normaliy assumed (cf. Alfvén, 1968). This can occur in several different ways, see e.g. Block and Fälthammar (1976). For instance, the behavioun of laboratony plasmas has led people to think in terms of current-ariven plasma instabilities, producing double layers (e.g. Block, 1972; Swift,1975) or anomalous resistivity (e.g. Kindel and Kennel, 1971; Swift, 1965), as the means by which fieldaligned currents are obstructed in aunoral regions. Alternatively, the magnetic mirroring may play the key role in this respect, as demonstrated by knight (1973) and Lemaine and Scherer (1974). The magnetic mirroxing may, in principle, support a parallel electric field even in the absence of a field-aligned current, as discussed originally by Alfvên and Fätthammar (1963) and Persson $(1963,1966)$. See also Whipple (1976). 


\section{Essential Features of a New Model}

The model outlined in the present paper basically belongs to category (c), but important features of it are taken from (a) and (b). The basic line of thought is the following:

From curl $\bar{E}=0$ it is seen that an electrostatic potential gradient along the geomagnetic field lines will be associated with an increased peak amplitude of $E_{\perp}$ at high altitudes. In order for the high-altitude $E_{2}$ to stay at reasonable values the region with a parallel potential drop of several $\mathrm{kV}$ must have a fairly wide spatial extent transverse to the magnetic field, in accondance with the observed latitudinal thickness of "inverted-V" events, which is typically $100-300 \mathrm{~km}$. This means that thin auroral precipitation structures like auroral rays must be due to a local reduction of the parallel "resistivity", rather than due to a current-induced local increase of the resistivity. This problem may be solved in terms of the following model. The upward field-aligned portion of a magnetosphere-ionosphere current system will be associated with a depletion of ambient cold electrons at high altitudes and, hence, the upward current will be carried by downflowing hot and dilute magnetospheric electrons: As the parallel motion of these electrons is strongly hampered by the magnetic mirroring a large fraction of the total voltage produced by the magnetospheric "dynamo" will be projected along the magnetic field, increasing both the flux density and the kinetic energy of the downflowing hot electrons. The effective "resistivity" may then be locally reduced either by a local increase of source electrons of by a local transfer of electron gyroenergy into electromagnetic wave-energy, for instance. In this manner very thin substructures of increased precipitation may occur, energized by the parallel electric field, that is in reality by the magnetospheric "dynamo". 
This paper emphasizes the nerits of this model in explaining a lange variety of observed phenomena, but it does not discuss the detailed physical conditions for the formation of thin precipitation structures.

\section{Some observational Indications of a Lange $(\Delta V)$}

The observation traditionally considered as indicative of field-aligned potential gradients is that of a "nearly monoenergetic" peak in the energy spectrum of precipitating electrons (Albert and Lindstrom, 1970; Arnoldy et al,1974; Choy et al, 1971; 0'Brien and Reasoner, 1971; Westerlund, 1969).

The frequently observed collimation (along $\vec{B}$ ) of auroral electron distributions is often interpreted in texms of a field-aligned acceleration at low altitudes which may be due to a $(\Delta V) \|$ (Ackenson and Frank, 1972; Arnoldy et al, 1974; Bosqued et al, 1974; Hoffman and Evans, 1968; O'Brien and Reasoner, 1971; Whalen and McDiarmid, 1972). Detailed measurements of pitch-angle versus energy have also led to an interpretation in terms of field-aligned potential gradients (Arnoldy et al, 1974), or even more specifically in terms of double layers (Albert and Lindstrom, 1970 ).

The large amount of satelifte data on "inverted-V" preaipitation structures (e g, Frank and Ackenson, 1971) and associated irregularities in the convection electric fieid (e.g. Cauffman and Gurnett, 1972) observed in the poleward part of the auroral ovals also seem to point in a direction of potential gradients along the magnetic field. These observations will be more extensively discussed in the Section "Comparison with observations". A direct evidence fon a lange $(\Delta V)$ || above an auronal form has been found recently from the drift motion of a barium plasma jet injected along the magnetic field ines (Wescott et al, 1976). While the barium plasma beyond $I R_{E}$ experienced 
flux tube splitting and rapid dispersion the plasma at lowen aItitudes nemained unaffected.

\section{Magnetic-Field Aligned Cuments}

Among the different theories of auroral parcicle acceleration, those involving parallel electric fields seem to be particularly encouraged by the existence of magneticfield aligned currents. This can be seen by the following arguments .

According to the viewpoints (a) and (b) Iisted in the Introduction, the auroras are in fact produced by a magnetospheric electron gun. The negative change camied by the electron beam need not give rise to a ret fieldaligned current, however, because the negative charge thus deposited deep within the ionosphere will enable ionospheric electrons in the topside region to escape outwands along the beam (together with backscattered and secondary electrons). Actually, this is what we should expect according to the kinetic model discussed by Lemaine and Scherer (1973a and 1974). In this way a net fieldaligned current may not appear until the precipitation flux density exceeds the flux density of freely escaping ionospheric electrons, which is quite high, maybe as high as $10^{11} / \mathrm{cm}^{2} \mathrm{~s}$ (or more) at ionospheric altitudes (cf Lemaire and scherer, 1973a and 1974). In the absence of precipitated negative charges the outflowing ionospheric electrons are tied to the much slower positive ionospheric ions (ambipolar diffusion).

The situation is quite different when we consider the viewpoint (c). A potential gradient along magnetic field Iines in a direcrion to accelerate precipitating electrons is an efficient barrien to the upward escaping thermal electrons from the ionosphere (actually a potential barrien of only a few volts will do, cf Lemaire and Scheṛer, 1973a and 1974) 
as well as a barrier to backscattered and secondary electrons. Consequently, if the flux of precipitating protons is negligible as compared with the electron flux (as is normally the case, of e.g. Frank and Ackerson, 1971), the net $i_{1}$ has to be in the upward direction and at least as large as the current dersity carried by the precipitating electrons (not including the downflowing electrons that have been reflected by the potential bamier).

On the other hand, if the net $i_{\mid}$is found to be at least as large as the precipitation current density, the most immediate interpretation is that the ionosphere, at the point of deposit of negative charge, stays at a positive potential of at least a few volts relative to the adjacent magnetosphere. This potential distribution is easy to understand if the precipitating electrons are passive carriers of current. However, as discussed in Section 8 the energetic precipitating electrons do not readily act as passive current carriers, unless they are forced to by an $e(\Delta V) \mid$ which is at least of the same onder of magnitude as the kinetic energy of the electrons. The reason for this is the magnetic mirroring in the geomagnetic field. $A(\Delta V)$ | of this magnitude will evidently appreciably increase the energy of the electrons at the same time.

The permanent existence of east-west extended field-aligned current sheets in a.l. local time sectors of the auroral ovals is fairly well established (Armstrong and Zmuda, 1970; Zmuda et al, 1970; Zmuda and Armstrong, 1974). It is interesting that an upward $i_{\mid}$(downgoing electnons) measured by means of its distortion of the geomagnetic field is very often seen directly associated with precipitating electrons and auroral aros and that the current density is seemingly at least as high as defined by the precipitation flux density (Anmstrong et al, 1975; Annoldy et al, 1974; Berko et al, 1975; Choy et al, 1971; Cloutier et al, 1973; Park and Cloutier, 1971). 


\section{Some Crucial Properties of a Large $(\Delta V)$ |)}

When discussing magnetospheric electric fields in the light of the behaviour of laboratory plasmas, we have to consider the entirely different boundary conditions in the magnetosphere. Fon an illustration of this, see Figure 1. The left part of the figure, $a$, is a rough sketch of the familiar electric field generated at a resistant section of an otherwise good conductor, when a current is flowing. The medium outside of the conductor is assumed to be a vacuum. The conductor in Fig la may be an unmagnetized plasma colurn contained wihtin a glass tube, and the "resistant" section may be a potential double layer (cf Block, 1975). The plasma confinement may as well be due to an external axial magnetic field.

Consider now Fig Ib. This is a sketch of the formal magnetospheric analogue of Fig la. For simplicity the total magnetic field (geomagnetic field and superposed field due to the current) is assumed to be vertical and homogeneous, $B \equiv B_{z}$. A current is flowing upward along $\vec{B}$, carried by downflowing electrons and having a density $i_{z^{*}}$ This current is obstructed by a region of reduced parallel "conductivity" oll. The word "conductivity" here simply means the quantity $i_{z} / E_{z}$

In the magnetospheric case thene is obviously no vacuum outside of the current path. Hence, the fan-shaped equipotentials in Fig la transform into magnetic-field-aligned equipotentials as indicated in Fig 1b. Suppose that the wavy contour in Fig $1 b$ indicates the smallest possible box containing the region with $E_{z} \neq 0$. Evidently, $E_{x}$ is not confined to the immediate vicinity of the box as in Fig Ia but penetrates infinitely far out along $\bar{B}$.

Further assume

$$
\operatorname{curl} \overline{\mathrm{E}}=0
$$


If we take the line integnal of $\overrightarrow{\mathrm{E}}$ around any closed contour and apply Stoke's theorem to Eq. (I) we get $\hat{\Phi} \overline{\mathrm{E}} \cdot \mathrm{d} \overline{\mathrm{S}}=0$

With refenence to Fig. Ib this can be written as

$$
\left|E_{x}\left(z_{2}\right)-E_{x}\left(z_{1}\right)\right|_{\max } \simeq\left|\frac{V\left(z_{1}\right)-V\left(z_{2}\right)}{\Delta x}\right|_{\max }
$$

Hence, if we know the extreme values of the transverse electric field $E_{1}$ at high altitudes, we also know the highest possible values of $(\Delta V[x]) \|^{/ \Delta x}$, where $(\Delta V) \|^{\text {is }}$ the potential drop along $\bar{B}$. That is, the larger $(\Delta V)||$ is, the larger is $\Delta x$.

This result can easily be generalized to a case with a dipolar total magnetic field where $E_{\text {II }}$ is arbitrarily distributed along a magnetic field line. If we integrate the longitudinal component of (1) along a magnetic field line from the equatorial plane down to the ionosphere, utilizing orthogonal dipolar coordinates (cf e.g. Cummings et $\underline{\text { al }}, 1968$ ), we get after some algebra:

$$
\left(\frac{\partial}{\partial s_{\perp}} \Delta V_{\mid}\right)_{i a}=E_{I_{i a}}-\frac{R^{2} e p}{R_{i a}^{2}} f(\theta) E_{I_{e p}}
$$

The symbol $E_{\perp}$ is used here for the southward component of the transverse electric field. The index "ia" denotes an ionospheric altitude and "ep" the equatorial plane. $R$ is the respective radial distance from the centre of the earth. $\Delta V_{\mid l}=V_{e p}-V_{i a}$ and $s_{1}$ is the southward horizontal

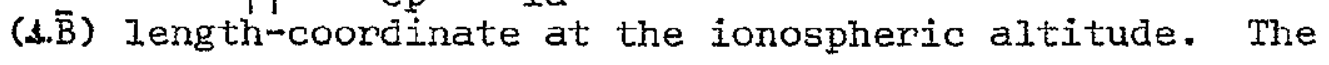
symbol $\theta$ is the magnetic co-latitude of the ionospheric intersection of the field line, and

$$
f(\theta)=\left(1+(1 / 4) \tan ^{2} \theta\right)^{1 / 2}\left(2 \sin \theta \cos \theta+(1 / 2) \tan \theta \sin ^{2} \theta\right)^{-1}
$$

that is, $f(\theta) \simeq 0.7$ with $\theta \simeq 20^{\circ}$. 
As a consequence of Equations (2a) - (2c) we may face an unattainable compromise when trying to apply a particular model of a current-dxiven plasma instability to the magnetospheric problem. This is because on one hand the instability may require a high value of $i_{z}$, while on the other hand (2a) - (2c) require the parallel electric fiejd to be spread out, and the capability of the ionosphere to carry horizontal currents is Iimited:

$$
\bar{i}_{1}=\sigma_{\mathrm{p}}\left(\overline{\mathrm{E}}_{\perp}+\bar{v}_{\mathrm{n}} \times \overline{\mathrm{B}}\right)+\sigma_{\mathrm{H}} \overline{\mathrm{B}} \times\left(\overline{\mathrm{E}}_{\perp}+\bar{v}_{\mathrm{n}} \times \overline{\mathrm{B}}\right) / \mathrm{B}
$$

The symbols $\sigma_{\mathrm{p}}$ and $\sigma_{\mathrm{H}}$ denote the Pedersen and Hall conductivities, respectively, and $v_{n}$ denotes the neutral gas velocity (cf Boström, 1964).

If we integrate (3) with respect to altitude, we get the total current per meter that can be fed into the ionosphere by means of a field-aligned sheet-curxent density $I_{\|}$. Roughly speaking, we may equate for instance $\Sigma_{p} E_{\perp}$ to $2 \Delta x$ i $\|$, where $\Sigma_{p}$ is the height integrated $\sigma_{p}$, and $\Delta x$ is defined by Fig Ib. The largest possible value of $\Sigma_{p}$ is probably about 40 mho (Boström, 1964). According to satellite measurements (Cauffman and Gurnett, 1972) $E_{2}$ at $500-2500 \mathrm{~km}$ alt is at most $150-200 \mathrm{mV} / \mathrm{m}$, which would thus imply $I_{\|}=\Sigma_{\mathrm{p}} E_{1} \leqslant 8 \mathrm{~A} / \mathrm{m}$. However, this is probably far too much, because $E_{3}$ tends to be reduced where $\Sigma_{p}$ (and $\Sigma_{H}$ ) is large (Aggson, 1969; Potter, 1970; Wescott et al, 1969). A mone realistic maximum value - of $I_{1}$ is nather an order of magnitude lower, in accordance with the magnetic measurements made by zmuda et al, 1970. These authors infer the values $0.02-0.7 \mathrm{~A} / \mathrm{m}$ for $I_{\|}$. The highest values of $i$ /| ever reported are about $2 \times 10^{-4} \mathrm{~A} / \mathrm{m}^{2}$ (Whalen and MoDiarmid, 1972). Using this value for $i||$ and $0.7 \mathrm{~A} / \mathrm{m}$ for $I_{\|}$we get $2 \Delta x \sim 3 \mathrm{~km}$. That is, with $V\left(z_{1}\right)-V\left(z_{2}\right)=5 \mathrm{kV}$ in Eq. (2b) we have $\left|E_{x}\left(z_{2}\right)-E_{x}\left(z_{1}\right)\right|-3 \mathrm{~V} / \mathrm{m}$. Hence, we may, for instance, conclude that any instability that does require $i||$ of at least $10^{-4} \mathrm{~A} / \mathrm{m}^{2}$ to be operating in the topside ionosphere is highly unlikely to be the actual cause of a $(\Delta V) \|$ of several $\mathrm{kV}$. 
From Eq. (2b), for instance, we further conclude that the common ray structure in visible auroras cannot be directly related to the spatial (transverse to $\bar{B}$ ) distribution of $(\Delta V) \|$. In fact, the ray structure might indicate $\Delta x<50 \mathrm{~m}$ in Fig. Ib (cf Chamberlain, 1961). With a typical electron kinetic energy of $5 \mathrm{keV}$ the right-hand member of $\mathrm{Eq}$. (2b) is thus $100 \mathrm{~V} / m$. That is, neglecting $E_{x}\left(z_{1}\right)$ we have $E_{x}\left(z_{2}\right)>100 \mathrm{~V} / \mathrm{m}$. In a dipolar magnetic field this would correspond to $E_{\perp}>40 \mathrm{~V} / \mathrm{m}$ at an altitude of $I R_{e}$ above the ionosphere and $E_{1}>1.5 \mathrm{~V} / \mathrm{m}$ in the equatorial plane at a distance of $10 \mathrm{R}_{e}$ from the earth (cf Eq. (2c)). These values of $E_{1}$ are at least a facton of 100 to 300 largen than the largest $E_{\perp}$-field observed so far (cf Caufman and Gurnett, 1972; Jeffries et a1, 1975; Wescott et al, 1976). Even if $E_{i}-f_{i e l d s}$ of this magnitude do occur they do not automatically imply that $(\Delta V)_{y}$ is confined within a very thin flux tube, however. The confinement of $(\Delta V)_{\|}$also requires that these strongly concentrated $E_{\perp}$-fields are supported by the magnetospheric dynamo (of. Section 14), which appeans rather

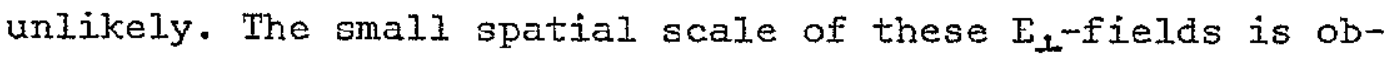
viously much smaller than the gyroradius of a $5 \mathrm{keV}$ proton, for instance. Hence, if the auroral electrons do indeed fall through a lange electrostatic potential gradient the auroral ray most likely represents a substructure within a much widex region of non-zero $E_{1}$. It should be noted that even though Equation ( 1 ) may not be strictly fulfilled, a region of large $(\Delta V) \|$, which is confined within a thin magnetic fiux tube, in general has to be associated with large values of $E_{\perp}$.

In summary, the magnetospheric case may require a large $(\Delta V)$ II to be maintained at current densities that are too Iow to generate a current-driven instability. The mechanism. responsible for $(\Delta V)||$ may, however, allow local enhancements of the precipitation within the region of non-zero E $\left.\right|^{\text {. As }}$ will be discussed below the magnetic mirroring may seem to provide a basic solution of these problems. 


\section{A Simple Illustration}

The simple model presented below will senve as a further illustration of the previous section. As our à priori information about $E_{\perp}$, we will assume a simplified horizontal distribution that is basically similar to the $E_{1}-$ distribution seen at auroral latitudes by a polar orbiting satellite at around $500-2500 \mathrm{~km}$ altitude, of for instance Cauffman and Gurnett (1972).

Fig 2 refers to the local evening side of the nowthen auroral zone. The z-axis points vertically upward, the $x$ - and $y$-axis point geomagnetically northward and westwand, respectively. Again the total magnetic field $\overline{\mathrm{B}}$ is assumed to have straight and vertical field lines.

Suppose that we know the convection at altitude $z_{b}$ in the upper ionosphere to be magnetically eastward and westwand, that is parallel to the $y$-axis, with $E_{2}$ equal to the step function $E_{x}\left(x, z_{b}\right)$ in Fig 2. What will $E_{x}$ then look like at other altitudes? Suppose that $\sigma_{\|}=i_{z} / E_{z}$ is constant above a certain altitude $z_{b}-h$ well above the E- and $F-$ layers, and that $\sigma \| \sim \infty$ throughout the lower part of the ionosphere. The last assumption enables us to get the height-integrated form of Eq. (3) by simply writing $\Sigma_{p}$ and $\varepsilon_{\mathrm{H}}$ instead of $\sigma_{\mathrm{p}}$ and $\sigma_{\mathrm{H}}$, provided that

$$
v_{n} \approx 0
$$

The Pedensen and Hall conductivities are assumed to be horizontally homogeneous.

Further assume that

$$
\frac{\partial}{\partial y}=0
$$

We now solve Eq. (1) with $E_{x}\left(x, z_{b}\right)$ and $E_{q} .(3)-(5)$ as boundary conditions, recalling that

$$
\operatorname{div} \bar{i}=0
$$


As the Pedersen current in this geometry has a divergence around $x=0$ we get a field-aligned current flowing upward at the field reversal. Due to the finite $\sigma / 1$ the step in $E_{x}\left(x, z_{b}\right)$ is completely smoothed out in $E_{x}\left(x, z_{b}-h\right)$ giving a finite $i_{z}$. In flying through the field reversal at altitude $z_{b}+h$ we would see an $E_{\perp}$ profile according to $E_{x}\left(x, z_{b}+h\right)$ and at even higher altitudes the "spikes" on each side of the reversal would be even larger.

We see from Fig 2 that, although the field-reversal is infinitely sharp at $z_{b}$, we get a certain finite characteristic thickness of the associated field-aligned current sheet equal to $2 h$, where

$$
\Lambda=\sqrt{\frac{h \cdot \Sigma_{p}}{\sigma / 1}}
$$

That is, to get $\Lambda=0$ we must have $\sigma \|=\infty$ everywhere. This is due to the fact that $E_{I}$ has a finite amplitude at $z_{b}$ (as well as all other altitudes).

It is important to notice, that as $\sigma_{\|}$decreases (increasing 1) the potential drop along the magnetic field is reached at the expense of the transverse potential drop at low altitudes, provided of course that $E_{I}\left(z_{b}\right)$ does not increase. In Fig. 2 $(\Delta V) \|_{\mid}$between $z_{b}-h$ and $z_{b}$ evidently has a maximum of $\Lambda \cdot E_{0}$ at $\mathrm{x}=0$.

We further notice that the parallel electric field $E_{z}$, as well as the current density $i_{z}$, has an "inverted-v" profile. This is of great interest, as this electric field having an upward direction on the evening side of the earth, at a "regular" field reversal, would be able to accelerate auroral electrons downwards, producing the typical "inverted-V" shape of mean energy versus latitude that is seen at the field reversal at local evening (Gumett and Frank, 1973). This is further discussed in Section 14. 


\section{A Self-Consistent Model of a Static E/I}

Figure 3 is a refinement of Fig. 2 in that the field-aligned "pesistivity", that is, the quantity $E_{z} / i_{z}$, is increased only within the field-aligned current sheet. In Fig. 3 $z_{b}$ is $1500 \mathrm{~km}$ and $\mathrm{h}=500 \mathrm{~km}$. The assumed boundary value $E_{x}\left(x, z_{b}\right)$ is not a step function here but a smooth profile, of the curve labeled $z=1500 \mathrm{~km}$. The altitude-averaged parallel field $\tilde{\mathrm{E}}_{z}$, defined. by,

$$
\tilde{E}_{z}(x)=\left(V\left(x, z_{b}-h\right)-V\left(x, z_{b}\right)\right) / h
$$

has been introduced here, as well as the altitude averaged "anomalous resistivity" $\tilde{\rho}_{\|}$that is

$$
\tilde{p}_{\mid}=\tilde{E}_{z} / i_{z}
$$

(the field-aligned current is assumed divergence free above the $\mathrm{E}-$ and $\mathrm{F}$-layers). Below $z_{\mathrm{b}}-\mathrm{h}=1000 \mathrm{~km} \tilde{\rho} \|$ is assumed small (nomal) in accondance with most theoretical nodels (e.g. Block, 1972; Kindel and Kennel, 1971).

The function $\tilde{\rho}||(x)$ defined by the bottom curve in Fig 3 is simply an $a$ priori assumption about the quantity $\tilde{E}_{z} / i_{z}$ (the peak value $10^{2} \mathrm{ohm} \cdot \mathrm{m}$ is chosen to give a reasonable current density). This assumption together with Eq (3) - (5) with $\Sigma_{P}=10$ mho has been used to solve $E q$ (1) and (6) for $\tilde{E}_{z}(x), i_{z}(x)$ and $E_{x}(x, z)$. The resulting distribution of $i_{z}(x)$, the top curve of Fig. 3 , then also defines $\tilde{\rho}_{\|}$as a function of $i_{z}$. This enables a better assumption about $\tilde{\rho}_{\|}$, which can be used to necalculate $i_{z}$, and so on. By this iterative process it is possible to get the desired relation between $\tilde{\rho}||$ an $i_{z}$.

The dashed $E_{x}$-profiles in Fig. 3 show what $E_{\perp}$ would look like at $\mathrm{z}=2000 \mathrm{~km}$ and $2500 \mathrm{~km}$ in case $\tilde{\rho} / /$ had the same average value throughout this upper altitude region. 
This again illustrates the fact that the amplitude of $E_{\perp}$ has to be an increasing function of altitude near the reversal due to the presence of a strong $E_{\mid l}$. Of course, this will only be true up to a certain altitude, because $(\Delta V)||$ is finite. Above this altitude we expect $E_{1}$ to be small ( in general) and the amplitude of $E_{1}$ to be decreasing with increasing altitude, due to the diverging magnetic field lines.

Despite the obvious difference between the two models, the quantity $\Lambda$ according to (7) again gives a good measure of the latitudinal width of the Ë-profile in Fig. 3 with the peak value $10^{2} \mathrm{ohm} \cdot \mathrm{m}$ inserted for $\sigma \|^{-1}$. Also, the maximum potential drop between $z=1000 \mathrm{~km}$ and $z=1500 \mathrm{~km}$ is again at least of the same order of magnitude as $\Lambda$ - $E_{0}$.

In Fig. $3 \Sigma_{p}$ is still horizontally homogeneous, but an assumed profile $\Sigma_{p}(x)$ can be introduced in the model to give a modified $i_{z}$ and $E_{z}$, which then helps make a better assumption about $\Sigma_{p}(x)$. Provided that the energy of the electrons before acceleration by $(\Delta V)_{\|}=h \cdot \tilde{E}_{z}$ is specified. In this way the model can be made self-consistent. The main effect of a locally enhanced $\Sigma_{p}(x)$ at the base of a field-aligned-cument sheet, when $\sigma_{\|}$is small and $E_{\perp}$ is given at a certain high altitude, is just a corresponding local reduction of $E_{\perp}$ at low altitudes, as has been shown in a previous paper (Lennartsson, $1973 \mathrm{a}$; cf also Section 14). The local increase of $\Sigma_{p}$ will also have a feed-back effect on the magnetospheric "dynamo", which this simple model cannot account for, however. Neither can this model in an adequate manner account for the auroral electrojet, which requires a three-dimensional geometry.

It may be noted that if we try to extend this simple model to very high altitudes (utilizing (2c), for instance) we can no longer neglect the (unknown) transverse current that is feeding the current loop (the "dynamo current"). 


\section{8. "Anomalous Resistivity" and the Magnetic Mixsoring}

Suppose for a moment that we can neglect the mirroring effect of the geomagnetic field where E $/ 1$ is large. Consider Fig. Ib. It is quite obvious that an electron that has gained the energy e $\left(V_{1}-V_{2}\right)$ by falling from $z_{2}$ to $z_{1}$ at the same time has made its maximum possible contribution to a short-circuiting current. That is, a freely falling electron in this case represents an extremely high conductivity per particle. In onder for the potential jump $V_{1}-v_{2}$ to be maintained, the number flux of these electrons thus has to be Iimited in one way on another. It is commonly assumed that the ionospheric and magnetospheric plasmas are dense enough to short-circuit any $E_{1}$ in the classical sense, but due to the high relative drift velocity between electrons and ions, some plasma instability is generated that may strongly limit the number flux of electrons, in analogy with the behaviour of laboratory plasmas. The main different modes of plasma instabilities that are considered in this respect are turbulent wave-particle interactions (e.g. Kindel and Kennel, 1971; Swirt, 1965) and electrostatic potential double layers (e.g. Block, 1972 and 1975; Kan, 1975; Swift, 1975). However, as discussed in Section 5 the magnetospheric plasma may not provide the appropriate boundary conditions for this kind of instability. The problems seem to be particularly severe with respect to turbulent "resistivity". In this case the electrons falling from $z_{2}$ to $z_{1}$ in Fig. Ib may possibly be a minority group of either "runaway" electrons on hot magnetospheric electrons passing by unaffected by the wave field, but the theory does require a certain minimum drift velocity of the themal electrons. That is, with an assumed plasma density the theory does require a minimum parallel current density being carried by thermal electrons i|l (thermal). 
Consequently, a certain minimum fraction of the electrostatic energy (provided by an extemal "dynamo") being released per unit area per second, $i_{l} \cdot\left(v_{1}-v_{2}\right)$, has to be converted into random motion, that is into heat (Block, 1975). With at least i $\|$ (themal) $\sim 10^{-5} \mathrm{~A} / \mathrm{m}^{2}$ (Kindel and Kennel, 197I) $\mathrm{v}_{1}-\mathrm{V}_{2}=5 \mathrm{kV}, \mathrm{z}_{2}-\mathrm{z}_{1}=10^{4} \mathrm{~km}$ (see Fig. Ib) and a density of thermal electrons and ions of $10^{9} / \mathrm{m}^{3}$, we get a heating rate, of $30 \mathrm{eV}$ per second per themal particle, to be compared with the original themal energy of less than $I$ eV per particle (cf Block, 1975). A certain fraction of this enengy may possibly be radiated away by plasma waves, but there is anyway no theory disproving that the auroral plasna' temperature would get drastically increased, as fan as the author knows (in the theory by Buneman, 1959, this heating is a desired effect; of also Biskamp and Chodura, 1973). We notice here that as the temperature is increased the maintaining of the plasma drift instabilities demand a higher i|l, which means that we immediately get into conflict with section 5 , even if the initial conditions are favourable.

The theory of double layers, on the other hand, does not suffer from this heating dilemma, according to Block (1972, 1975), because the particle motion is basically laminan at a double layer. The theory of double layers will not be discussed in the present paper, however. The reader is referred to the papers by Block (1972, 1975) as well as the papers by Swift (1975) and Kan (1975) on oblique double layers (electrostatic shocks).

Apart from the heating problem there is also a problem of plasma depletion connected with any theory that requires a strong (upward) ill to be carried by a cold background plasma of ionospheric origin. Suppose for instance that the density $n$ of coid electrons at an altitude of $3000 \mathrm{kms}$ is $10^{2} / \mathrm{cm}^{3}$ (cf Lemaire and Scheren, 1973b) and that $\mathrm{n}$ decreases proportionally to $\mathrm{B}$ upwards, giving $\mathrm{n} \sim 0.1-1 / \mathrm{cm}^{3}$ at the 
equatorial point of the magnetic field Iine. This may well give an overestimation of the cold-plasma content in a typical flux tube, as mass spectrometen data (Chappel1, 1972) frequently show a total ion density of only $0.1 / \mathrm{cm}^{3}$ in the plasma sheet. An upward field-aligned current with $i|| \sim 10^{-5} \mathrm{~A} / \mathrm{m}^{2}$ at topside altitudes, camried by downward-moving electrons, would deplete all available cold electrons above $3000 \mathrm{kms}$ altitude within about $2-5$ minutes (provided that the current sheet does not have a persistent horizontal motion relative to the plasma). Within a few moxe minutes even the copside ionosphere would suffer a strong depletion. If $i_{1}$ as high as $10^{-4} \mathrm{~A} / \mathrm{m}^{2}$ is requined by the theory, the depletion problem gets extremely critical. We cannot really expect the cold plasma to be supplied from adjacent flux tubes by convection, as the convection is generally found to be along auroral arcs (e.g. Armstrong et al, 1975; Gurnett and Frank, 1973; Wescott et al, 1959). At i ll as high as $10^{-5}-10^{-4} \mathrm{~A} / \mathrm{m}^{2}$ we can also neglect the contribution from upward-moving protons, even though these can, in principle, be continously supplied by the topside ionosphere. This proton current cannot be stronger than allowed by the escape flux $\left(10^{-7}-10^{-6} \mathrm{~A} / \mathrm{m}^{2}\right.$, cf Lemaire and Scherer, $1973 a$ and 1974), that is, it is "temperature-limited". The "spacecharge-Iimited" proton current is even many orders of magnitude weaker (Block, 1967). ConsequentIy, the only available carriens of a persistently intense upward i / ane the hot electrons from the outer magnetosphere.

Now take into account the magnetic mimroring effect within the acceleration region. The previous statement about the high "conductivity" of "freely falling" electrons does not hold true anymore because the magnetic mirror tends to obstruct the parallel current by deviating the parallel motion of the electron into transverse motion (without changing the particle energy). Apparently, this may even constitute the only necessary mechanjsm fon obstructing 
ill and maintaining a lange total potential drop along the geomagnetic field lines, provided that the density of the charge carriers is not too large. In view of the fact that an upward current (downgoing electrons) may have to be camied mainly by electrons from the dilute and hot plasma in the outer magnetosphere, this mechanism seems to have been somewhat overlooked over the years.

Consider the simplified case with an isotropic and monoenergetic source distribution of electrons in the outer magnetosphere, at point 1 . In this case the electron flux density at ionospheric altitudes, at point 2 , is proportional to $\sin ^{2} \alpha_{\max }$, where $\alpha_{\max }$ is the maximum pitch angle at point 1 of electrons capable of reaching point 2 . If the potential difference between points $I$ and 2 is $(\Delta V)_{\|}>0$, the constancy of the magnetic moment gives

$$
\sin ^{2} \alpha_{\max }=\frac{B_{1}}{B_{2}}\left(I+\frac{e(\Delta V)||}{\frac{\mathbb{R}_{e}}{2} v_{I}^{2}}\right)
$$

where $B_{1}$ and $B_{2}$ are the magnetic field strengths at points 1 and 2 , respectively, and $\left(\mathrm{m}_{\mathrm{e}} / 2\right) \mathrm{v}_{1}{ }^{2}$ is the electron kinetic energy at point 1 . Hence, the field-aligned current density at ionospheric altitudes due to precipitation is given by

$$
i_{\|_{2}}=n_{1} v_{1} \frac{e}{2}\left(I+\frac{e(\Delta v)}{\frac{m_{e}}{2} v_{1}^{2}}\right)
$$

where $n_{1}$ and $v_{1}$ are the density and velocity of downgoing electrons at point $1 . \mathrm{Eq} .(8)$ is valid for $e(\Delta V) \| \leq$ $\lesssim\left(m_{e} / 2\right) v_{1}^{2} B_{2} / B_{1}$. For larger $(\Delta V) \|$ the current becomes saturated. If the electrons at point 1 have a Maxwellian distribution, for instance, Eq. (8) takes on a different algebraic form (Knight, 1973), but (8) is still a good approximation, with $v_{1}$ denoting an average value. As a numeric example, with $2 \mathrm{n}_{1}=0.1 \mathrm{~cm}^{-3}$ and $\left(\mathrm{m}_{e} / 2\right) \mathrm{v}_{1}{ }^{2}=500 \mathrm{eV}$, a cumpent density i $\|_{12}$ of $10^{-6} \mathrm{~A} / \mathrm{m}^{2}$ requires $(\Delta V) \| \Rightarrow \mathrm{keV}$. 
It may be noted that the $(\Delta V) / /$ required to drive a certain high $i_{\mid / 2}$ will increase with increasing energy (at constant density) of the source plasma, as long as $n_{I} e v_{I}<i_{1 / 2}$

Knight (1973) and Lemaire and Scherer (1973a, 1974) have used more sophisticated models to calculate the relation

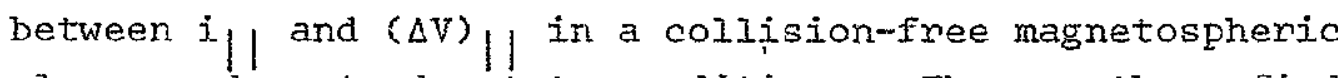
plasma under steady state conditions. These authors find, by assuming reasonable ionosphere-plasmasheet parameters, that an upward $i \mid l$ of the order of $10^{-5} \mathrm{~A} / \mathrm{m}^{2}$, for instance, may readily require a total $(\Delta V) \|$ of $I-10 \mathrm{kV}$, as a result of the magnetic mirroring of plasmasheet electrons (see for instance Fig. 3, p. 745, in the paper by Knight). Knight has neglected the ionospheric ion contribution to i||, while this is included by Lemaire and scherer, but the difference is obviously insignificant when $i|| \geqslant 10^{-5} \mathrm{~A} / \mathrm{m}^{2}$.

Unfortunately, the model by knight provides only the total voltage $(\Delta V) \|_{\text {(as does }}$ (aq. (8)) while $E_{\|}$) remains undetermined. The model by Lemaire and Scherer does provide E numericaliy but the case of a strong upward $E_{\|}$is not explicitly shown.

It should be noted that these models rely upon the outer magnetosphere providing a steady supply of isotropic electrons. The need for a high $(\Delta V) \|$ will evidently be langer if the magnetospheric particle source is depleted of electrons with small pitch angles. A second important point that has to be considered is that the outflux of positive ions from the ionosphere may become strongly reduced by spacecharge effects when the cold electrons are depleted at high altitudes (cf Block, 1967). 


\section{The Transverse Spatial Distribution of $E_{\|}$and the Ray Structure of Auroras}

In view of Sections $5-7$ it may seem difficult to reconcile auroral fine structures like visible auroral rays (and very thin current sheets) with a particle acceleration due to a large $(\Delta V) \|$. This is particularly true of currentdriven instabilities as we then would expect the crosssectional dimensions of a current sheet or current beam to directly map the transverse dimensions of the $(\Delta V)||^{-}$
region, of Fig. $1 \mathrm{~b}$. This may thus strongly favour the magnetic-mirroring as the actual current obstructing mechanism, according to the following arguments.

As seen above the magnetic mirror may support a large $(\Delta V) \|$ even at a small current density. Eq. (8) assumes an isotropic distribution of source electrons at point 1 , however. If the source electrons alI have large pitch angles $(\Delta V)$ || may be high even with no net $i_{\|}$being, carried by the hot electrons ( $C f$ Alfvên and Fälthammar, 1963, PP 162 - 167; Persson, 1963 and 1966). Given the potential difference $(\Delta V)$ | along a certain field line the parallel current density i/f may thus vary over a wide range with different density and pitch-angle distribution of the source electrons. We therefore suggest the following basic model.

By the magnetic minroring of incoming electrons the hot plasma at high altitudes remains at a large negative potential relative to the cold plasma at the ionospheric end of the flux tube (see Section 14 for the driving "dynamo"). The resulting parallel electric field is distributed within a high-altitude region that may have large dimensions transverse, as well as parallel, to the magnetic field, and net field-aligned currents may be flowing preferably in thin field-aligned subregions.

This model is compatible with the requirements in Section 5 , and it permits thin auroral rays to be energized by $a(\Delta V) \| \cdot$ 
What remains is a mechanism for producing thin precipitation structures. It is conceivable that such a mechanism may be associated with the supplying of electrons at high alticudes, but the above model also offers the possibility of transforming a uniform source distribution of electrons into a non-uniform distribution of precipitating electrons. That is, a local increase of the precipitation may, in principle, be accomplished by a local recuction of the magnetic mirroring effect on electrons. Hence, with the above model it may be possible to reduce the problem of auronal ray formation to the problem of finding a waveparticle interaction by which electrons can lose gyroenergy in a spatially selective manner. This problem will not be further analyzed here, however, but it may be kept in mind that there are, in fact, a number of plasma wave modes already known that resonate with the gyromotion of electrons (see e.g. Helliwell, 1967; Kennel and Petschek, 1966; Perkins, 1968; Stix, 1962). It should be noted that this kind of wave-panticle interaction does not necessarily have to occur

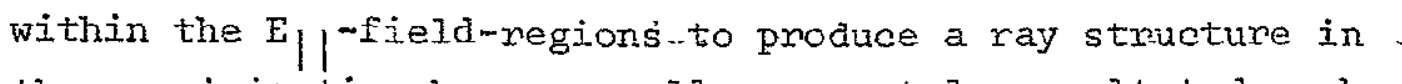
the precipitation but may, well occur at lower altztudes where $E_{||}=0$, if the magnetic mirror ratio is still large below.

\section{The Parallel Spatial Distribution of $E_{1} / \mid$}

The distribution along the magnetic field of $E_{1}$ in a magnetic mirron configuration is a problem that has been treared in certain aspects by, among others, Persson (1966). He finds, for example, that in a stationary state with no field-aligned currents and with "almost isotropic" distributions (isotropic except for a loss cone) for both electrons and ions the parallel electric field is given by

$$
E_{\|} \propto \operatorname{grad}_{\|} \cdot \mathrm{B}
$$

This particular form of $\mathrm{E}_{\|}$will be used in Sections 11 and 13 for quantitative calculations. The results obtained there are qualitatively true with a rather wide range of $E_{t^{\prime}}$ fields, however. Although $(9 a)$ was derived by 
Persson in a case with no net i be compatible with a large $i \mid f$ as well, at least in a restricted sense. In fact, $(g a)$ is the unique solution of $\mathrm{Eq} .(8)$ under the following conditions.

Suppose Eq. (8) is valid at any point 2 along the magnetic field Iine below point $I\left(B_{2}>B_{1}\right)$. This implies that the electrons are monoenergetic with an isotropic pitchangle distribution on the interval $\left[0^{\circ}, 90^{\circ}\right]$ at every point below point 1 . Then if $s$ is the length-coordinate along the field line, Eq. ( 8$)$ is differentiable with respect to $s$ at point 2. Since $i_{\|}$(s) $\propto B$ (s) the derivative of (8) gives

$$
E_{||}=-k \frac{d B}{d s}
$$

with $k=\mu_{\text {crit }} / e, \mu_{\text {crit }}$ being the magnetic moment of the electrons that maintain a pitch angle $\approx 90^{\circ}$.

Hence, (9b) is the electric field that barely maintains a saturated current at every pont, given an isotropic and monoenergetic source distribution of electrons at point 1 with energy equal to $\mu_{\text {crit }^{B}}{ }_{1}$. If the electrons at point 1 have a distribution of energies the electric field ( $9 \mathrm{~b})$ provides an asymptotically saturated current as $B(s) / B_{1} \rightarrow \infty$.

The actual distribution of $\mathrm{E} / \mathrm{l}$ is subject to quasi-neutrality of the plasma as well as to the magnetic mirroring of the charge camriers, however. In the problem considered by Persson Eq. (9D) is consistent-with quasi-neutrality, but only trapped particles are present $\left(\mu_{e}\right\rangle \in k$ ). In the auronal case the distribution of $E_{\|}$is necessarily influenced by the ionospheric particles. Consider Fig. 4. Suppose the high-altitude region $I$ is devojd j of cold electrons and $E_{\|}$is defined by ( $9 a$ ) in this region. Suppose further that the cold plasma still remains in the low-altitude region 3 , where the plasma density $n_{3}$ is much larger than the plasma density $n_{1}$ in region $1, n_{3} \gg n_{1}$. In region 3 the electric fiela (9a) cannot be valid and we assume ${ }^{\prime}||=0$ throughout 
this region. The intermediate region 2 is a transition region with some à priori unknown distribution of $E / \mid$ In going from the low-altitude region 3 into the E/ffield at higher altitudes the cold protons (or other positive ions) $\mathrm{P}_{3}$, rapidiy decrease in density and soon become comparabie in density with the hot protons $P_{I}$. In region 1 the two proton populations $P_{1}$ and $P_{3}$ together may thus (chiefly). match the negative charge due to the hot electrons $e_{1}$. At lower altitudes in region 3 the hot electrons $e_{1}$ are a minority group and the protons $p_{3}$ are matched by cold electrons there. In the intermediate region $2 E_{\|} \neq 0$, however, and no cold electrons can exist where E/ fovo. The only negative particles that can conceivably match the protons $\mathrm{p}_{3}$ throughout region 2 are the backscattened and energetic secondary electrons $e_{3}$ from below. Hence, the transition region 2 either has a quasi-neutral plasma, with a density gradient defined by the energy distribution of the backscattered and secondary electrons or it has an unbalanced positive charge in the lower section. The formen case allows a smooth density gradient while the latter case obviously requires a steep density gradient, defined by some characteristic Debye length, and a locally strong $E_{\|}$. The latter case also requires a region of unbalanced negative charge above the positive change. This negative charge may be partly due to the electrons $e_{3}$ and partly due to energetic electrons $e_{2}$ that have become trapped between region $I$ and the magnetic mimror below during the initial growth of $(\Delta V) \|$. Both the gradual transition and the "doubie layer" are conceivable, but the "double layer" may seem the most likely since it does not require any particular velocity distribution of the electrons (Block, private communication). It should be noted that such a "double layer" is not a result of a strong current, but is a result of the magnetic mirroring of the curnent carniers.

From these considerations $i t$ is obvious that the parallel electric field cannot be entirely determined by the local magnetic field gradient at every point along the flux tube. 
Below some altitude, which may vary with time, the electric field will be "screened out" by the cold plasma still remaining there. This also means that there has to be a transition region where $E_{\mid}$is determined by local plasma parameters, as well as by the magnetic mirror ratio below the transition region. At least if this mimron ratio is large the partial potential difference $(\delta V) / \mid$ across the transition region may be a considenable fraction of the total potential aifference $(\Delta V)$, cf. Fig 4 . At higher altitudes the E//field may or may not be able to adjust to the local magnetic-field gradient, depending on the actual particle population. However, even if $E_{\mid}$does not have a smooth distribtuion like ( $9 a)$, for instance, it still has to extend to high enough altitudes to ensure a sufficient precipitation flux of source electrons, according to Eq. (8). This problem with be discussed further in a forthcoming paper.

\section{The Colimation of Electron Bunsts}

Suppose $E_{\|}$is given by ( $(B)$ ) with $k$ being a positive constant along a certain field line. The equation of motion along the field line for an electron is then

$$
m_{e} \frac{d v}{d t}=(e k-\mu) \frac{d B}{d s}
$$

where $\mu=m_{e} v_{2}^{2} / 2 B$ is the magnetic moment. In going from point $I$ where $B=B_{1}$ to point 2 where $B=B_{2}>B_{1}$ an electron increases its total kinetic energy according to

$$
\frac{m_{e}}{2}\left(v_{2}^{2}-v_{1}^{2}\right)=e\left(v_{2}-v_{1}\right)=e k\left(B_{2}-B_{1}\right)
$$

As long as $\mu$ is constant we then have

$$
\frac{v_{12}^{2}-v_{11}^{2}}{v_{2}^{2}-v_{I}^{2}}=\frac{u}{e k}
$$


That is, if $B_{2} \gg B_{1} \quad\left(v_{12}>v_{11}\right)$

$$
\sin ^{2} \alpha_{2}=\frac{v_{12}{ }^{2}}{v_{2}{ }^{2}} \lesssim \frac{\mu}{e k}
$$

Hence, given an electron with $\mu$ < ek its pitch angle remains smaller than or equal to a constant determined by the magnetic moment of the electron. Such an electron increases its parallel velocity along the path, according to (I0), and the increase is faster the smaller $\mu$ is. The associated velocity despersion due to different a at given energy is quite lange. Suppose for instance that ( $3 b$ ) is valid down to an altitude of $h=0.5$ earth radii above the earth on a magnetic field line with $I=10$ and $(\Delta V) \|_{\|}=1 \mathrm{keV}$. If electrons with $10 \mathrm{eV}$ energy stant at $\mathrm{L}=10$ in the equatorial plane, an observer at altitude $h$ will find a time delay of about 4 sec between the arrival of electrons with $\alpha=0^{\circ}$ and $\alpha=45^{\circ}$. An observer in the lower ionosphene will find this time delay to be only $I$ sec, if $(\delta V) \mid=0$ below $h$, but this is more than one order of magnitude langer than would be expected if the electrons stanted from altitude $h$ with $1 \mathrm{keV}$ energy. A sudden increase of the number of electrons with $\mu<$ ek at high altitude is then expected to show up as a burst of electrons with small pitch angles at lower altitudes, in agreement with auroral particle observations (e. g, Arnoldy et al, 1974; O'Brien and Reasoner, 1971).

An increase of the number of electrons with $\mu<$ ek does not have to involve an actual particle injection, it may as well be accomplished by a transfer of electrons in velocity space from large to small magnetic moments. That is, a gyroresonant wave-particle intexaction may be a solution in this case, too. A transfer of electron gyroenergy into electromagnetic wave enengy may also, in principle, produce a collimated bearn of electrons by itself, without involving velocity dispersion. If the interaction occuns within the electric field ( $9 b$ ) the field serves to preserve the collimation, according to $\mathrm{Eq}(12 \mathrm{~b})$. 
These considerations are based on the exact relation ( $9 \mathrm{~b})$, but they are essentially applicable to any upward $E$ that is distributed along the magnetic field with $e \|_{\mid}$ds $\mu \Delta \mathrm{B}$ over finite intervals $\Delta s$;

\section{Comments on the Electron Energy Spectrum}

Considen Eq. (11). If the initial energy at point $I$ is small compared to $e\left(V_{2}-V_{1}\right)$, only a small total amount of kinetic (gyro) energy has to be removed by wave-particle interactions at point 1 to create a iarge enhancement of the elctron energy fIux at point 2. On the other hand, any electron that is being mirroxed at a point close to point 2, between points 1 and 2 , can lose all its gyroenergy and arnive at point 2 virtually without any energy. In other words, as long as we do not specify in detall the process of waveparticle interactions, there is a wide range of possible precipitation spectra associated with any given total potential drop $(\Delta V) \mid{ }^{\prime}$

Obviously, thene may also be certain wave-particle interactions connected mainly with the parallel motion of the electrons (cf Stix, 1962). Since the precipitating electrons will be streaming through a population of upflowing positive ions from the ionosphere there may be favourable conditions for the two-stream instability, for instance. The different kinds of possible wave-particle interactions thus seem likely to generate a component of the precipitating electrons having a degraded energy, as compared with the "free-fall" component. More generaliy, wave-particle interactions may cause the electrons to diffuse in velocity space, preferably towards smaller velocities, although some precipitating electrons may gain energy in this way (cf Perkins, 1968). As the backscattered and numerous secondary electrons will be reflected downwards by $(\Delta V) \|$ (Evans, 1974) the total energy spectrum of precipitating electrons at low altitudes may thus, in principle, look fainly smooth even with a large $(\Delta V) \|$ present, which may also be the case with a fluctuating $(\Delta V)_{\mid}$. 
The extent of energy degradation of the primany electrons will probably be a function of the spatial structure of the precipitation, as indicated by straightforward calculations (cf for instance Hess et al, 1971). That is, a widely extended structure is seemingly particularly susceptible to instabilities in velocity space because it allows unstable traveling waves to grow for extended periods of time. By the same token a spatially strongly limited plasma may thus be mone stable against this kind of wave growth. In fact, both artificial generation of thin "auroral" electron beams (Hess et al, 1971) and theoretical investigations (Jones and Kellogg, 1973) do indicate that a thin structure of precipitating electrons is very stable to energy degradation by wave-panticle interaction. This finding has an important implication with respect to the present auroral model. Suppose the number of electrons with $\mu<$ ex is increased in a narrow spatial region at high altitudes, by gyroresonant wave-particle interaction, for instance. When continuing downwands these electrons will form a thin structure of increased number density (consider Eq. (11) and nV ||$\sim B$ ) that may thus tecome stable against further energy degradation, leading to a bright auroral form and a pronounced "monoenergetic" peak in the energy spectrum (cf O'Brien and Reasoner, 1971; Westerlund, 1969). That is, in principle the "monoenergetic" peak in the electron energy spectrum may have a spatial. (horizontal) fine-structure within the horizontal region covered by a large $(\Delta V) \|^{\prime}$

\section{A Generalized "Loss Cone" for the Electrons}

Since an electron with a large magnetic moment will mirror, there may often be a tendency towards negative charge-accumulation on a closed field line with upward cumrents. This may tend to quench the magnetospheric dynamo current (of next section) leading to a "loss cone" distribution of electrons. This "Ioss cone" will be larger for electrons having a smaller total energy than for the more energetic electrons. Suppose ${ }^{E} / /$ is given by ( $9 \mathrm{~b}$ ) above a centain altitude, called the 
altitude of penetration of $E_{\|}$, where $B=B_{\text {pen }}$, and - $E_{\|}=0$ below. Then, given a point on a closed magnetic field line, where the magnetic field strength is $B \leq B_{\text {pen }}$, the half-angle $\alpha_{1 e}$ of the local' loss cone is defined by

$$
\sin ^{2} \alpha_{1 e}=\frac{B}{B_{\max }}+\frac{e \kappa B}{\frac{\mathrm{e}_{e}{ }^{2}}{2}}\left(\frac{\mathrm{B}_{\mathrm{pen}}}{\mathrm{B}_{\max }}-\frac{B}{B_{\max }}\right)
$$

where $B_{\max }$ is the magnetic field strength at the altitude of the low ionosphere. For $B_{\text {pen }}<B \leq B_{\max }$ the constant $k$ is zero. No electrons with $m_{e} v^{2 / 2} \leq e k B\left(B_{p e n}-B\right) /\left(B_{\max }-B\right)$ will remain on the field line. Eq. (13) follows from Eq.(1I) and the constancy of $\mu$.

The corresponding equation for the proton "loss cone" is analogous to (13) with the plus sign changed to a minus sign. This is then applicable to protons with $m_{p} v^{2} / 2 \geq \operatorname{ex}\left(B_{p e n}-B\right)$. Protons with lower energies will have no "loss cone".

If the low-altitude region where $B \geq B_{\text {pen }}$ has an upward $E_{1 \mid} \neq$ with a large $(\delta V) \|$ associated with it the change of Eq. (13) may be approximated by a reduction of $B_{\text {max }}$, $B_{\max } \rightarrow B$ pen . In the case of the protons $B_{\max }$ will effectively increase.

If the electric field is increased, that is if $k$ is increased to $K^{\prime}>k$, electrons will start precipitating again with pitch angles given by

$$
\frac{\kappa}{k} \leq \sin ^{2} \alpha \leq I
$$

at the altitude of the low ionosphere. The half-angle of the apparent "loss cone" at ionospheric altitudes will thus rapidly decrease from $90^{\circ}$ to the lower limit determined by (14) and then slowly increase again. Provided that the electric field has a negligible growth time, the time scale of the initial decrease of this "loss cone" will be roughly defined by the time it takes an electron with a low initial 
energy to fall from an altitude of one earth radius above the altitude of penetration of $E_{||}$, that is typically of the order of a second or less. The time scale of the subsequent increase of the "loss cone", on the other hand, will be detemined by the travel time from the outer magnetosphere of the lowest-energy electrons, which may be several tens of seconds (with initial energies of the order of $100 \mathrm{eV}$ on less).

Under certain conditions the increase of $E_{\text {| }}$ will lead to a transiently field-aligned precipitation flux which has a hardening energy spectrum towards smaller pitch angles, as observed at low altitudes. Consider, for instance, the case $K^{\prime} \gg x$ ( $K$ may be zero). Suppose the average electron energy at any altitude is much smaller than ex'B ${ }^{\prime}$ pen prior to the increase of $\mathrm{E}_{\|}$. After the electric field is "turned on", a given electron from the precipitating population will reach the low ionosphere at a smaller pitch angle and a higher energy, if it is initially at a higher altitude, as seen from Eq. (11) and the constancy of $\mu$. Furthenmore, as a consequence of the velocity dispersion an electron from a higher altitude (higher final enengy) will reach the low ionosphere with a certain pitch angle at the same time as an electron from a lower altitude (lower final energy) with a langer pitch angle. This apparent relation between energy and pitch angle will thus gradually disappear as electrons from larger and larger distances appear at $90^{\circ}$ pitch angle. Since a largen $(\Delta V)$ || will cause a larger number of electrons to precipitate, the velocity dispersion will also be associated with a fieldmaligned number flux at low altitudes. It should be kept in mind that the effect of velocity dispersion may be very strong with an electric field that is distributed over a lange distance (cf section 11). Also in the case of gradually increasing $(\Delta V)$ | the velocity dispersion will have similar effects, as discussed in Section 15. These results are basically true with any $E / \mid$ that is both distributed along the magnetic field line and compatible with preserved magnetic moments of the electrons. 


\section{The Magnetospheric Dynamo}

The current configuration of Fig. 2 and 3 nay be part of current loops like those sketched in Fig. 5. The view of Fig. 5 is in the direction towards the sun. We may think of Fig. 2 and 3 as pictures of a small region around point $D$. In this particular case Pedersen currents are flowing in towards the upward current sheet both from the north and the south side. The poleward downward curnent may flow either from $A^{\prime}$ to $D^{\prime}$ or from $W$ down the dotted line (that is, close to A-D), on probably both ways. This symmetric situation may not at all be the typical case in reality, where we may even have multiple field-aligned current sheets (e.g. Aubry et al, 1972).

A magnetospheric dynamo driving a current down to the ionosphere and back up basically is a continuously progressing charge separation in the outer magnetosphere, driven by e. $g$. inertia forces on the charged particles. The dashed current $100 p$ in Fig. 5 , for example, may be accomplished by the ionosphenic drag (in a hydromagnetic sense) on the solar wind flow via open (merged) magnetic field lines. This case is a "voltage-generator", where the charge separation between $A$ and $A^{\prime}$ is produced, basically, by solar wind protons displacing their gyrocenters in a direction opposite to (and the electrons in the same direction as) $\bar{E}_{1}$, when entering a region of reduced $E_{\perp}$ (reduced $E \times B$-drift), cf e.g.AIfven (1975). That is, kinetic energy associated with $\mathrm{E} \times \mathrm{B}$-drifting solar wind protons (and electrons) is converted into electrostatic energy. If the solid current loop in Fig. 5 is on closed magnetic field lines the driving change separation between $A$ and $B$ may, for instance, be due to gradient$B$ drift of energetic particles across inhomogeneities in their density and temperature distributions, as in the model by Jaggi and Wolf (1973). This case is more like a "currentgenerator", although far from strictly. Indirectly, the solan wind is the driving agent in this case, too, as the solar wind flow is what causes the internal magnetospheric 
convection (sunward). This convection carries the charged panticles into the earth's magnetic field, leading to betatron and Fermi acceleration of the particles (of e.g. AIfvén and FäIthammar, I963).

Consider, for instance, the current loop ABCDA in Fig 5 . Suppose the dynamo current $A-B$ is due to a differential drift of hot electrons and protons (ox other positive ions) across the magnetic field lines. This drift tends to accumulate electrons at $A$ and protons at $B$. Then the positive. space charge at $B$ will enable the ionospheric electrons at point $C$ to escape at a higher rate than allowed by the normal polar wind flux of both ions and electrons. Since the Ilux density of freely escaping ionospheric electrons is as high as $10^{10}-10^{11} / \mathrm{cm}^{2} \mathrm{~s}$, corpesponding to $i_{1} \sim 10^{-5}-10^{-4} \mathrm{~A} / \mathrm{m}^{2}$ (of Lemaire and Scherer, 1973), it is reasonable to believe that the current $B-C$ typically flows without a significant potential drop. That is, point $C$ will remain at nearly the same potential as point B. A Pedersen current from $C$ to $D$ in the jonosphere will thus charge point $D$ positive with respect to point $A$. The negative space charge at $A$, on the other hand, does not release a corresponding increase in the outflux of positive ions from point $D$. The upward positive-ion flux from the ionosphere will be eithen "temperature limited" by the ratumal themal outflux of topside ions, giving a contribution to $i_{1}$ of only $10^{-7}-10^{-6} \mathrm{~A} / \mathrm{m}^{2}$ (of Lemaire and Scherer, 1974) on "spacecharge limited", giving an even smallen contribution (cf Block, 1967). The potential difference $(\Delta V) \mid$ between $A$ and $D$ will thus easily gnow to a level where a large fraction of the hot electrons arriving at $A$ are forced through the magnetic mirron and precipitate at $D$ with increased energy. Given the initial (average) kinetic energy $K_{e}$ of the electrons at $A$, the magnetic mirror may support a $(\Delta V) \mid$ which is as lange as $\left(K_{e} / e\right) \times\left(B_{D} / B_{A}\right)$, where $B_{D}$ and $B_{A}$ are the magnetic field strengths at $D$ and $A$, respectively (see Section 8 ). This is valid provided most of the electrons from $A$ preserve their magnetic moments during transit to $D$, of course. 
By these arguments we emphasize the magnetic mirroring effect as the presumable basic cause of a large $(\Delta V) \mid \|^{*}$ That is, the primary effect of the minroring is to obstruct a discharge along the magnetic field line. However, the actual distribution of $E_{1}$ is subject to the quasineutrality of the plasma and this may well imposel the form mation of spacechange layers where $E_{j}$ i becones much stronger than the local mirron forces (cf. Section 10).

It may be noted that the acceleration conaitions along A-D' may also be influenced by changes in the magnetic energy stored in the current loop. This will not be discussed in this paper, however.

\section{Comparison with Observations}

The convection electric field in the altitude range 500 $2500 \mathrm{~km}$ has been extensively explored by polar orbiting satellites (Cauffman and Gurnett, 1972; Frank and Gurnett, 1971; Gurnett and Frank, 1973; Gurnett and Akasofu, 1974; Heppner, 1972 and 1973). It is found thac the convection is generally antisunward over the polar caps down to the poleward edge of the auronal ovals (at $70^{\circ}-80^{\circ}$ magnetic lat) and sunward between this region and the plasmasphere (except for stagnation lines near noon and midnight). The transition between antisunward and sunward convection is generally observed as a fairly sharp reversal of $E_{f}$, and adjacent to the main reversal the $E_{\text {f }}$ field is often strongly fluctuating (as seen by the moving spacecraft) with pronounced peaks. It has further been established (Ackerson and Frank, 1972; Burch et al, 1976; Frank and Ackenson, 1971; Gurnett and Frank, 1973) that on the evening side this field reversal is frequentIy associated with bands of intense "inverted- $v$ " events, that is field-aligned sheets (probably east-west oriented) of precipitating electrons characterized by an "inverted- $V "$ profile of mean energy versus latitude, as seen by a satellite crossing the sheet. From sections 6 and 7 we would expect such an "inverted-V" event associated with the field-reversal on the evening side, where the associated $i_{1}$ is upward, in contrast with the moming side (cf Fig. 5). 
The basic results of Sections 6 and 7 only require a pronounced horizontal gradient in $E_{1}$, that is, "inverted-V" events may as well be associated with the irregularities in $E_{1}$ adjacent to the main reversal, in accordance with the observations. The real situation, howeven, may be strongly complicated by horizontal gradients in $\Sigma_{p}$ (and $\Sigma_{H}$ ), in particular when the precipitation structure is moving. A furthen complication is introduced by the (generally) unknown neutral gas velocity, which may also slightly displace the "inverted-V" structure relative to the observed inhomogeneity in $E_{1}$ (cf Lennartsson, 1973a). That is, we might expect some ambiguity when interpreting the observations. As long as Pedersen currents are the major source of the upward $i_{\mid l}$ and the gradient in $E_{2}$ is mainly responsible for $i_{\|}$the $E_{\perp}$-gradient has to be in a certain direction, of course. That is, more $E_{\perp}$-field Iines have to point toward the upward current sheet than away from it. The occasional observation of "inverted-V" events in the fieldroversal region in the morning sector we thus interpret as due to the presence of a $\operatorname{grad}_{1} E_{1}$ with the "right" sign close to the main reversal. This seems to be in full agreement with data from the Ion Drift Meter (Hanson et al, 1973) on Atmosphere Explorer c, acconding to Burch (private communication).

We note from the simple models in sections 6 and 7 that the "inverted- $V$ " shape is the simplest possible latitudinal distribution of $(\Delta V) \|$ we may expect at an upward fieldaligned current sheet, when the parallel "conductivity" is finite.

The "inverted- $V$ " events in genenal are fairly thick sheets of precipitation, typically $100-250 \mathrm{~km}$ (Burch et al, 1976) which obviously is in full agreement with section 5 (cf Eq. (2b)). It is even observed that in the direction from early to late local evening the "inverted-v" precipitation bands grow more energetic and wider (e.g. Gurnett and Erank, 1973), which is in qualitative agreement with Eqs $(2 \mathrm{~b}-\mathrm{c})$ 
under the assumption that $E_{\perp}$ at lange distances from the earth (the "dynamo field") stays fainly constant; of also Eq. (7) and the two subsequent paragraphs in Section 6; note that a decreased "o/1" may, for instance, be due to an increased temperature of the hot source plasma, according to $\mathrm{Eq}_{\mathrm{f}}(8)$.

Often very pronounced peaks with opposite signs in $E_{1}$ are observed at each border of an "inverted-V" event. According to Burch (private communication) the ion drift data from Atmosphere Explorer C (Hanson et al, 1973) show such pronounced peaks to be in the "right" sense in all cases examined, that is with $E_{2}$ pointing towand the center of the "inverted-V" event. Besides, Burch found one pain of opposite $E_{\perp}$ peaks where $E_{\perp}$ pointed outwards, and this single case was seen to be associated with a three orders of magnitude dropout in the electron precipitation flux between the $E_{1}$-peaks as compared with the surrounding flux. Even if the satelite is observing the uppermost $E_{1}$ curve in Fig. 3, for instance, a large fraction of the observed $E_{\perp}$ is evidently due to the low-altitude field needed to carry the Pedersen currents towards the upwand current sheet. That is, we do not expect the integral of $E_{1}$ along the satelitite trajectory to be a true measure of the $(\Delta V)$ p below the satellite. The "asymptotic" $E_{\perp}$-field on both sides of the reversal in Fig. 2 and 3 in reality may well go to zero within a short distance from the reversal, associated with the downward $i_{\mid}$-sheets (upflowing ionospheric electrons) as sketched in Fig. 6. This means that the satellite may at times observe two apparent "spikes" in $E_{2}$ even if it is actually observing only the low-altitude $E_{2}$.

Observations indicate that $E_{\perp}$ is reduced within auronal arcs due to the enhanced $\Sigma_{\mathrm{p}}$ and $\Sigma_{\mathrm{H}}$ (e.g. Aggson, 1969; Potter, 1970; Wescott et al, 1969). This is in full formal agreement with a large $(\Delta V) \|$, as shown in an earlier paper (Lennartsson, 1973a), and may be qualitatively understood as the low 
altitude $E_{\perp}$ adjusting to gradients in $\Sigma_{p}$ to avoid a too-

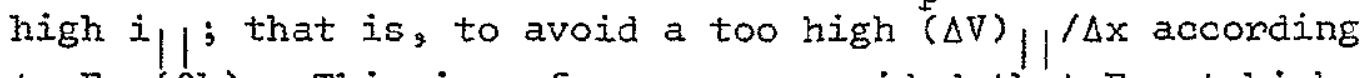
to $\mathrm{Eq}(2 \mathrm{~b})$. This is, of course, provided that $E_{2}$ at high altitudes (the "dynamo field") is not allowed to increase. This is also illustrated in Fig. 6 , where the low-altitude $E_{I}$ is very weak within the upward current sheet (precipitating electrons).

As mentioned in Section 4 observations often indicate that $i_{1 /}$ as inferred from magnetic measurements, is equal to or even langer than the current density inferred from the detected precipitation (see references at the end of section 4). We found (Section 4) that this is seemingly the same as keeping the ionosphere at a higher potential than the adjacent magnetosphere. Obviously, this is easily understood in terms of a large $(\Delta V) \|$ in a direction to accelerate electrons downwards (along $A-D$ in $F i g .5$ ).

The above comments apply in principle to any large $(\Delta V) \|^{\text {. }}$ Now consider some observations that may favour a combined model of magnetic mirroring and plasma waves.

We recall that the "inverted-V" stxuctures are often fainly wide in latitude and, hence, often associated with average $i_{1 /} \sim 10^{-6} \mathrm{~A} / \mathrm{m}^{2}$ (or less) at ionospheric altitudes (see references at the beginning of this section). This evidently places a severe restriction on a current-driven instability being the cause of $(\Delta V) \|_{\text {. }}$ on the other hand, the interpretation in terms of magnetic mirroring is, in principle, not affected at all, as $(\Delta V)||$ in this case will be determined by the overall supply of electrons with small magnetic moments provided the outflux of ionospheric ions is not too large,

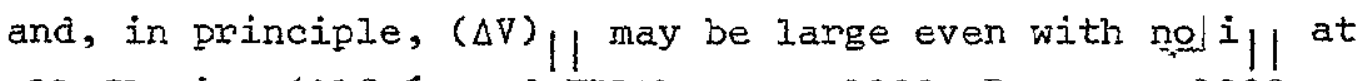
all flowing (Alfvên and Fälthammar, 1963; Persson, 1963; cf Section 13). With this model we then allow very sharp auronal arcs to be associated with intensified substructures of || within wider regions of weak (upward) i (cf Armstrong et al, 1975; HeIlinam and Davis, 1970). the intensification of $i_{\mid}$being due, for instance to 
Iocal reduction of the "magnetic resistance"; that is, due to local transfering of electrons from the dirroring population to the precipitating population by-means:of gyroresonant wavemparticle interaction.

According to Sharber and Heikkila (1.972) no systematic variation with altitude of the auroral particle enengies has been observed from a few hundred kms altitude to a few thousand kms. This is well compatible, within the accuracy of comparing observations of different events, with a widely distributed $E / \mid$. The most straightforward interpretation is, however, that $\mathrm{E}_{\mid y}$ does not domally penetrate to these altitudes.

We note that $E_{\|}$according to (9a), for instance; allows us to apply, in a qualitative manner, the model by Evans (1974), where he gives an explanation of the low-erengy "continuum" spectrum of auronal electrons in tems of secondary and backscattered electrons from the ionosphere being reflected downwards by a $(\Delta V) \|$. In Evans' model all primaxy electrons have energies equal to or larger than e( $\Delta V)$, (they have all fallen through the same potential ( $\Delta V) \|^{-}$) giving rise to a pronounced high-energy peak in the energy spectrum. According to the present model (see in particular Section 12) we may expect some of the electrons with lower energies, to be primary electrons that have been degraded in energy by wave-particle interactions. As the intensity and spectral shape of the backscattered and secondary electrons with lower energies are rather insesitive to the enengy of the primaxies (the intensity may even tend to increase with decreasing energy of the primaries, cf Evans, 1974) we might expect essentially the same low-enengy "continuun" if the primary high-energy peak is "smoothed out" by wave-particle interactions. Even outside the magnetic flux tubes with a high $(\Delta V) /$ there will be a residual precipitation, and if the magnetic field lines are closed we might then find a somewhat similar low-energy "continuum" 
of electrons arriving from the conjugate ionosphere. In this manner it may be possible to reconcile the presence of a high $(\Delta V) \|$ with the frequent observations of a fairly stable low-energy "continuum" even when the high-energy peak is strongly fluctuating ( $0^{\prime B r i e n}$ and Reasoner, 1971; Reasoner and Chappe11, 1973; Westerlund, 1969).

$A(\Delta V)||$ supported by magnetic mirroring will have a selective effect on a given source distribution of electrons incident at high altitudes. The electrons with lower initial energies have on the average smaller magnetic moments and will thus get more completely precipitated. As $(\Delta V)$ grows larger, more high-energy electrons will be precipitated, while the precipitation of the electrons with lower energies will become successively saturated. An observer going towards the center of an "inverted-V" event (cf Fig. 3) will thus see a progressively larger high-energy tail above the (increasing)

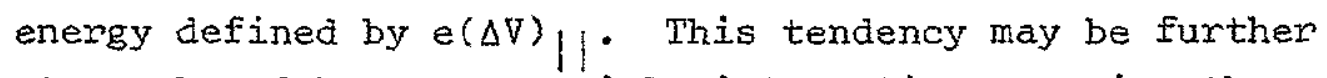
strengthened by wavemparticle interactions causing the precipitating electrons to diffuse in velocity space (cf section 12). Recent measurements (Burch et al, 1976) do show a similar behavioum of the electron energy spectrum at low altizudes.

As pointed out in Section 11, the common collimation of electron bursts to small pirch angles (Foffman and Evans, 1968; O'Brien and Reasoner, 197.I; Whalen and MCDiamid, 1972) may be understood in terms of a distributed E $E^{-f i e l d}$ in conbination with gyroresonant waves acting as triggers of bursts (rays).

Arnoldy et al, (1974) found field-aligned fluxes (bursts) of electrons to have distinctly lower peak energies than the accompanying isotropic and "monoenergetio" component. In at least one case even the field-aligned flux appeared to be "monoenergetic", although with a lower energy than the isotropic flux. It may be possible to explain these 
observations simply in terms of several electrons populations with different energies falling through a certain $(\Delta V) \mid$, at least if all limitations of the instruments are taken into account. However, we note that these observations fit into a model where bursts and rays are triggered by a transfer of electron gyroenergy into wave energy. Such an energy transfer leads to a reduced total energy of the colizmated electrons as compared with an undisturbed isotropic component. A "monoenengetic" beam of collimated electrons is conceivable as a result of electrons losing most of their energy, at least their gyroenergy, at a relatively well-defined altitude above the observer. At the same time, some electrons may lose energy by wave-particle interactions without getting effectively collimated (cf section 12); that is, we may not expect to find a very simple general relation among observed energies and pitch angles, particularly not if velocity dispersion is likely to be important (cf Whalen and MoDiarmid, 1972; of also Section 13).

Venkatarangan et al, (1975) have studied the electron pitchangle distribution within "inverted-v" structures by means of a spinning satellite (the low-altitude polar orbiting satellite Isis 2). They have frequently found the fluxes in all energy channels to peak at $90^{\circ}$ pitch angle, while the lower energy flux has shown a secondary peak at small pitch angles. The fact than only the lower energy electrons show an increased flux at small pitch angles we may again (in principle) ascribe to a wave-triggered collimation, which is basically associated with a certain loss of particle energy. An apparent peak flux at $90^{\circ}$ at all energies, as seen by the spinning spacecraft (with spin period $\sim 20 \mathrm{sec}$ ) looking in only one radial direction at a time, may possibly be a temporal or spatial variation in the flux. Alternatively, the observations may be due to "loss cone" distributions on closed field lines, as described in Section 13, with some electrons being scattered into the "collimation-cone" (small. magnetic moments) somewhere along the field-line, associated with energy 105 . 
Several authors (Dunckel et al, 1970; Gurnett, 1974; Kurth et al, 1975) have observed very intense bursts of electromagnetic "kilometric" radiation propagating away from the earth during the occurence of discrete auroral forms. By direction-finding technique (Gurnett; 1974; Kurth et al, 1975) the radiation source has been found to be located in the auroral zones, in particular in the local evening sector, at altitudes of about $1-2 \mathrm{R}_{\mathrm{e}}$ above the earth: The radiation spectrum has a peak intensity in the range $100-300 \mathrm{kHz}$, and the total instantaneous powen of the radiation from the earth has been estimated to be as lange as $1 \%$ of the maximum power dissapted by auroral particle precipitation (Gunnett, 1974). In view of the estimated altitude of the sounce the typical frequencies of this nadiation are obviously compatible with a wave generation at the local electron gyrofrequency, in particular if we consider the effect of Dopplen shifts. If auroral ray formation is indeed associated with a transformation of gyroenergy into wave energy this is obviously a kind of radiation we would expect.

Rosenberg et al, (1971) have observed a one-to-one correlation between buxsts of VLF emissions and sightly time-delayed short bursts of $x$-rays (due to bursts of precipitating energetic electrons) at Siple station, Antarctica ( $L=4.1$ ). From, among other things, the frequencies of the VLF emissions (center frequency $2.5 \mathrm{kHz}$ ) Rosenberg et al interpret these emissions as due to cyclotron resonance between the waves and enengetic electrons at the equator, by which the wave energy is created at the expense of the electron gyroenergy. This is the same kind of wave-particle intexaction as suggested in section 9. However, the electron densities of main interest in the model may be smaller than the equatorial density in this case $\left(\sim 10 / \mathrm{cm}^{3}\right)$.

Sharber and Heikkila (1972) have observed, using a spinning satellite (the polan orbiting satellite Isis 1 ), that the electnon flux in the polewand part of the nighttime auroral 
ovals is fpequently field-aligned with a hardening of the energy spectrum at small pitch angles (" $\Lambda^{\prime}$ structures). When the range of the pitch angle scan is sufficiently wide, a more or less empty "loss cone" may show up ("topless $\Lambda^{\prime \prime}$ structures). They reject parallel electric fields as being involved and interpret their observations in tems of Fermi acceleration on closed field lines. As thein observations seem to be directly related to "inverted-V" events, thein interpretacion is evidently incompatible with the above model. We are thus forced to suggest a different interpretation, aIthough this may be somewhat ambiguous. We first notice that a hardening of the electron energy spectrum with decreasing pitch angles to a certain extent can be attributed to the "loss cone" being larger for low energies than for high (of Fig.7, $\mathrm{P} 3406$, in the paper by Sharber and Heikkila). Such an energy dependent "loss cone" is suggested Dy Eq (I3). (Isis I has an apogee of $3522 \mathrm{~km}$ alt). A sudden increase of $E_{||}$will, transiently (for some. tens of seconds), lead to a narrowed empty cone, as suggested by Eq (14). In particular, if the increase in $E_{1 \mid}$ is relatively very strong, that is, like the case $\kappa^{\prime}>k$ in Section 13 ( $\mathrm{k}$ may be zero), we may well expect the velocity dispersion to initiaIIy (within the first few seconds) cause an apparent electron energy spectrum where the peak energy is increasing towards smaller pitch angles. At the same time, the electron number flux will evidently be generally field-aligned ( $c f$ Eq. (II) and (I2b)).

However, it may be angued that the spin period of Isis I $(20.4 \mathrm{sec})$ is somewhat too long to really permit the latter explanation of the increasing energy towards small pitch angles and the field-aligned number flux. We may thus be forced to assume that the spinning spacecraft is also moving through a spatial ("inverted-V") structure at the same time, or that the spacecraft is measuring a mainly temporal change in the elctron energy and flux. 


\section{- 41 ORIGINAI PAGE IS \\ OF POOR QUALITY}

We further notice that even a gradually increasing ( $\Delta V) \|$, which is distributed along the magnetic field line, may cause the most energetic electrons to have the apparently smallest pitch angles at low altitude. This is because the most energetic electrons will be the most recently accelerated at any time during the increase of $(\Delta V) \|^{\text {. }}$ This mechanism, basically due to different starting times at a given location, is an alternative to the mechanism in section 13, which is due to different initial particle Iocations after a given sudden increase of $(\Delta V) \|^{*}$ During the increase of $(\Delta V) \mid$ the velocity dispersion will also cause a field-aligned number flux. The time scales involved in the low altitude precipitation event will be determined both by the growth rate of $(\Delta V) \mid$, and the actual instantaneous distribution of $E_{\mid l}$ along the magnetic field line.

Effects that may be due to a temporal or spatial variation of $(\Delta V) \|$ are, of course, possible on both open and closed magnetic field lines, but the presence of a "loss cone" may seem to imply closed field lines.

The simultaneous observations of electron and (weak) proton precipitation, with even higher proton energies than electron energies, have often been used as an argument against any significant $(\Delta V) /$ (O'Brien, 1970; Sharber and Heikkila, 1972). For the case of the procons and electrons originating from different regions along a magnetic field-line, Block (1972) has shown this to be surmountable in terms of centain distributions of $E_{\mid l}$. We do not want to restrict the above model to this case, however, but rather allow protons and electrons to originate from the same regions. The protons that are observed precipitating along with the electrons thus have been decelerated by $(\Delta V)$ | on their way down. The measured proton distribution does not, however, have a simple bearing on the "typical" energy aistributions of protons in the source regions, as long as we do not know whether on not the charge-separation process (the "dynamo") in the outer magnetosphere is associated with energy disperm sion. At times a large fraction of the precipitating protons 
may also be previously accelerated ionospheric protons as discussed furthen down. With an auroral proton energy typicaliy at least as high as the electron energy (cf Sharber and Heikkila, 1972) we expect the proton number flux to be about $1 / 40$ times the electron number flux, or

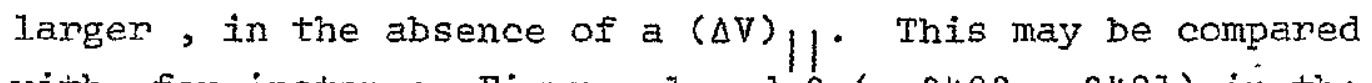
with, for instance, Figures 1 and 2 (p. 3400 - 3401) in the paper by Sharber and Heikkila (1972), where the peak number flux of electrons is apparently at least three orders of magnitude largex than the simultaneous proton number flux. This evidently means that the number density of the precipitating protons is much smallen than the number density of the precipitating electrons (the proton energy seems to be somewhat reduced, too). A simple interpretation of this is that only the protons from the high-energy tail of the source distribution are able to reach lower altitudes, due to the presence of a large $(\Delta V) \|$ in a direction to accelerate the electrons and retard the protons. The local quasineutrality may, of course, be maintained by upwand flowing ionosphexic ions. At the altitude of this particular obsenvation (around $3000 \mathrm{~km}$ ) upward accelerating ionospheric (topside) ions may have an energy of a few kev on less, depending upon the actual penetration depth of $E_{\|}$. However, in this particular case the spacecraft (Isis 1) was evidently looking in the upward direction only. The upflowing ionospheric ions will not readily be observed unless the observer is looking very close to the downward field-aligned direction (cf the discussion below).

According to Bunch et al (1976) the data from Atmosphere Explorer $C$ typically show the ratio of electron to proton energy fluxes to be strongly increased within "inverted-v" precipitation structures, as compared with the flux ratios outside. This is, of course, what we (generaliy) would expect if the electrons are being accelerated by a ( $\Delta V)||$. 
The present model leads, howeven, to anothen interesting consequence regarding proton precipitation or, more generally, precipitation of positive ions. The parallel electric field will evidently accelerate positive ions upward from the topside ionosphere (while suppressing the ionospheric electrons). These ions will, in principle, get an extremely field-aligned velocity distribution both as a result of the diverging magnetic field Iines and as a result of $E_{\mid l}$. If the magnetic field line is closed, these enengized ions may be able to precipitate in the conjugate ionosphere together with electrons, provided $(\Delta V)||$ is smaller along the downward path. Since these ions may drift a considerable distance transverse to the magnetic field during transit, for instance due to E X B - drift, they may be able to precipitate in either hemisphere if $(\Delta V) \mid$ has a horizontal gradient in the drift direction. A trapped population of these ions will precipitate whenever $(\Delta V) \|$ is reduced. That is, we may well expect energetic protons, as well as heavier ions (cf Shelley et al, 19723, of ionospheric origin to precipitate now and Then togethen with energetic electrons. The energy of these ions may occasionally be higher than the average electron energy, for instance when $\left.(\Delta V)\right|_{\mid}$is rapidly decreasing in time.

An interesting property of these ions is that they tend to have a field-aligned distribution also when precipitating at ionospheric altitudes. In fact, provided the magnetic moment is preserved, these ions will not be isotropic unless their energy is $\leqslant K_{0} \times B_{\max ^{\prime}} / B_{\text {pen }}$, where $K_{0}$ is the initial energy of the ions $\left(<1 \mathrm{eV}\right.$ ) before acceleration and $B_{\max }$ and $B_{\text {pen }}$ are the magnetic field strengths at the low altitude of observation and at the lowest altitude of penetration of $E_{||}$respeotively $\left(B_{\text {pen }}<B_{\max }\right.$ ). As a consequence, an observer at low altitudes will, on the average, find the fieldalignment to be more pronounced the highen the energy is of these precipitating ions. In this manner the present model may be able to give a very simple altennative explanation of the fairly frequent satellite observations (ESRO/A and ESRO/B) of field-aligned fluxes of positive ions in the 
keV-range along with energetic (and typically isotropic) electron precipitation (Hultquist, 1971), as far as these observations can be related to closed magnetic field ines. The field-alignment of these ions nay, to some extent, be reduced by wave-particle interactions, of course (cf section 12).

In the outer magnetosphere these energized ionospheric ions will presumably have a generally field-aligned distribution, even in the presence of a slight pitch-angle scattering, with energies of the same onder as the "typical" energy of auroral electrons or lower ( $(\Delta V) \| \leqslant$ typical electron energies) Furthermore, an $E_{\mid l}$ according to $(3 b)$; or more generally, any

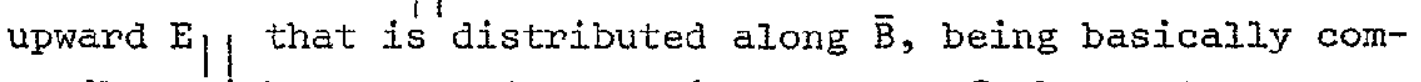
patible with preserved magnetic moments of the protons, will suppress the development of the loss cone of lowerm energy protons of magnetosphexic origin, on closed magnetic field lines (cf Section 13). This may explain the "sourcecone" distributions of positive ions at energies less than $10 \mathrm{keV}$ frequently found by the geosynchnonous satelitite ATS-6 (DeForest, private communication; McIlwain, 1975).

According to DeForest (private communication) and McIlwain (1975) even the electrons sometimes show strongly fieldaligned distributions at the geosynchronous orbit (ATS-6), although much less often than the positive ions. These field-aligned eleatron fluxes seem to be quite intense but rather fluctuating in amplitude (on a time scale of a quarter of a second, acconding to Deforest). The energy spectrum of these electrons is typically flat or slightly rising up to a break point somewhere between 0.1 and $10 \mathrm{keV}$, beyond which it rapidly decreases. I $\tau$ might be tempting to associate these electron "beams" with a downward current (upward moving electrons) iike B-C in Fig. 5. This would then require some kind of obstruction of the escape flux of ionospheric electrons (cf knight, 1973; Lemaire and Scherer, 1973a). One possible obstruction is a double layer (cf Block, 1972, 1975), anothen is strongly reduced plasma density at high altitudes, in which case the electron flux 
may become spacecharge-limited (cf Block, 1967). The latter case, for instance, may occur if the cold plasma at high altitudes has been previously depleted by a strong upward current (of Section 8). However, it may not be recessary to associate the electron "beam" with a downward curnent. Consider, for instance, un upward electric field according to $\mathrm{Eq}$. ( $\mathrm{Sb}$ ). If this electric field is "turned off" it will enable energized electrons from lower altitudes to escape outwards, after mirroring, while transforming thein gyromotion into directional (field-aligned) motion. Hence, if the total $(\Delta V) \|_{\mid}$is strongly fluctuating it may thus cause a fluctuating beam of field-aligned energetic electrons to appear in the outer magnetosphere. Such a fluctuating $(\Delta V)||$ is conceivable under certain conditions, since a current loop like ABCD in Fig. 5 is basically a "resonant circuit", the "inductance" being due to the encircled magnetic field and the "capacitance" being due to the changed-particle convection through the 100p (of Irennartsson, 1973b).

When the positive ions from the ionosphere reach the parallel electric field and become accelerated they also become reduced in density as compared with the density distribution during normal escape (for a review of the theory of escape flux, see e.g.the paper by Lemaire and Scherer, 1973b). Such a density reduction of the positive ions is also required to preserve quasi-neutrality, since the cold electrons will be depleted by the parallel electric field and replaced by a dilute population of precipitating, backscattered and (energetic) secondary electrons, of the brief discussion in Section 10. Without a detailed study of the quasi-neutrality we are very limited in predicting the plasma distributions above auronal forms, however. As a very general consequence of the present model we would, nevertheless; expect to find anomalousiy low plasna densities at high altitudes above auroral forms. This qualitative prediction may seem to be compatible with electron density measurements made by the 
topside-sounding satelitte Alouette II (Hagg, 1967). Ionograms recorded at high latitudes ( $L \geq 6$ ) and high altitudes $(1500-3000 \mathrm{~km}$ ) often showed a beat-frequency modulation from which Hagg deduced accurate densities as low as $8 \mathrm{~cm}^{-3}$ in many cases.

It is worth noting in this context that also the downwand field-aligned portion of a current loop, like ABCD in Fig. 5, may be associated with a plasma depletion, although in this case the depletion will occur at low altitudes. If the horizontal current $C-D$ is a Pedersen current it is associated with a transport of positive ions away from point $\mathrm{C}$ (of Bostrom, 1964). Since the cumrent $B-C$ will be associated with an outflux of electrons form point $C$ the plasma density at point $C$ may thus become appreciably reduced. This mechanism mey seem to have potential applications to centain observations of density irregularities in the high-latitude ionosphere but it will not be further discussed here. For a theoretical discussion of plasma depletion by field-aligned currents, see Block and Falthammar (1968).

\section{Summary and Concluding Remarks}

The existence of field-aligned currents associated with auroral precipitation (Section 4) suggests a process of charge separation (a "dynamo") in the distributions of "hot" particles in the outer magnetosphere and the magnetospheresolar wind transition region (Section 14), leading to current loops like $A B C D$ in Fig 5, for instance. The downwand current $B-C$ may generally be carried by escaping ionospheric electrons, changing point $C$ positive to essentially the same potential as point $B$. A Pedersen current $C-D$ will charge point $D$ positive relative to point $A$. However, the upward current D-A will generally have to be camried by down-flowing "hot" magnetospheric electrons (Section 8), which do not readily flow because of the magnetic mirroring (Section 8). This may lead to a lange $(\Delta V)||$ along $A-D$, entirely du to adiabatic particle motion, that will increase the number flux of precipitating electrons as well as the kinetic energy (Sections 
8-10). At the same time, this $(\Delta V) \|$ will energize outflowing positive ionospheric ions (Section 15). A region of high ( $\Delta V)$ /| generally has to be rather wide in latitude, as well as longitude, (Section 5), which requires an additional mechanism for producing auroral fine structures. For this reason, we suggest, as one plausible mechanism, a wave-particle interaction by which the downflowing electrons can lose gyroenergy. Any such interaction that is spatially localized will automatically lead to a locally intensified precipitation, like an auroral ray (Section 9 ). If this interaction occurs at high altitudes, within the

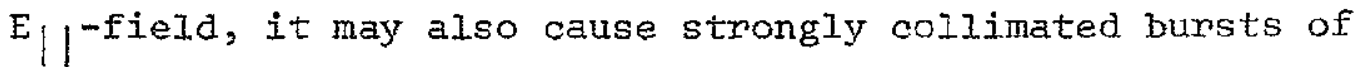
precipitation (Section 11). An alternative mechanism would have to involve a corresponding spatial fine structure in the electron source at high altitude.

In this model the parallel electric field, that is in reality the "dynamo", provides the increased energy flux in auroral. displays, whereas the spatial structure of individual auroral forms may be due to a modulating effect of certain kinds of plasma instabilities. These plasma instabilities may not provide "anomalous resistivity", however. On the contrary, these instabilities may just as well tend to limit the growth of $(\Delta V) \mid$ by thein themalizing effect on the otherwise-adiabatic particle motion.

The association of a net field-aligned current with precipitating electrons also suggests that the convection electric field $E_{I}$ is (genenally) transverse to auroral ares (cf observations made by Gumett and Frank, 1973, and Wescott ex al 1969) with steep gradients within the precipitation structure (Sections 6 and 7), particularly at altitúdes of several thousand kms (of Fig. 3 and Eq. (9a)). The resulting $E_{1} \times$ Bdrift pattern is likely to lead to different kinds of shear flow instabilities, similan to the Kelvin-Helmholiz instability, that may generate folds and vortex-forms (cf Haerendel, 1974; Hallinan and Davis, 1970). The frequent alignment 
of thin auroral forms along the $E_{\perp} \times$ B-drift direction (cf e.g. Hallinan and Davis, 1970) hence might indicate that the auroral ray formation is closely related to such shear flow instabilities.

It may be noted that the parallel electric field in this model is due to a magnetic confinement of a negatively charged, hot and collision-free plasma. A transfer of electron gyroenergy into wave-energy obviouśly tends to weaken the confinement; and if this energy transfer becomes too strong, the parallel potential gradient will break down. Hence, from this model, in contrast with centain other models of parallel electric fields, we might expect only a small fraction of the total auroral particle energy to be transformed into electromagnetic wave-energy during the acceleration process. According to observations, the auroral precipitation is indeed associated with electromagnetic radiation of many different wavelengths, but the radiated powen is seemingly small compared to the power carried by the precipitating particles. The most intense radiation discovered so far is the kilometric radiation, which still represents only approximately 1 percent of the total power dissipated by the auroral electrons (Gurnett, 1974). 


\section{Acknowledgements}

The author acknowledges many fruitful discussions with Drs. J. L. Burch, C. R. Chappell, S. E. DeForest, D. L. Reasoner and G. R. Swenson of the Marshall Space Flight Center, Huntsville, as well as Dr. L. P. Block and Prof. C.-G. Fälthamman of the Royal Institute of Technology, Stockholn.

The main part of this work was carried out while the author was an NAS/NRC Resident Research Associate at the George C. Marshall Space Flight Center.' The support from NRC and MSFC is gratefully acknowledged: 
Ackerson, K. L. and I. A. Frank, Correlared Satellite Measurements of Low-Energy Electron Precipitation and Ground-Based Observations of a Visible Auroral Arc, J. Geophys. Res., 77, 1128, 1972.

Aggson, T. L., Probe Measurements of Blectric Fields in Space, in Atmospheric Emissions, ed. by B. M. McCormac and A. Omholt, p. 305, Vam Norstrand Reinhold Co., New York, 1969.

Albert, R. D., and P. J. Iindstrom, Auroxal-Particle Precipitation and Trapping Caused by Electrostatic Double Layers in the Ionosphere, Science, I70, 1398, 1970.

Alfvén, H., The second approach to cosmic electrodynamics, Ann. Geophys., 24, 1, 341, 1968.

Alfvên, H., Blectric current structure of the magnetosphere, in B. Hultqvist and L. Stenflo (eds.), Physics of the Hot Plasma in the Magnetosphere, Plenum Press, Net York, pp. 1-2., 1975.

Alfvên, H., and C.-G. Fälthamenar, Cosmical Electrodynamics, Fundamental Principles, 2nd Edn., Oxford University Press, London, 1963.

Armstrong, J. C., S.-I. Akasofu, and G. Rostocker, A Comparison of Satellite Observations of Birkeland Currents With Ground Observations of Visible Aurora and Ionospheric Currents, J. Geophys. Res., 80, 575, 1975.

Armstrong, J. C., and A. J. Zmuda, Field-Aligned Current at $1100 \mathrm{kn}$ in the Auroral Region Measured by Satellite, J. Geophysp. Res., 75, 7122, 1970.

Arnoldy, R. L., P. B. Lewis, and F. O. Isaacson, Field-Aligned Auroral Electron Eluxes, J. Geophys. Res., 79, 4208, 1974.

Aubry, M. P., M. G. Kivelson, R. L. McPherron, C. T. Russe11, and D. S. Colburn, Outer Magnetosphere near lfidnight at Quiet and Disturbed Times, J. Geophys. Res., 77, 5487, 1972.

Berko, F. W., R. A. Hoffman, R. K. Burton, and R. E. Holzer, Simultaneous Particle and Field Observations of Field-Aligned Currents, J. Geophys. Res. , 80, 37, 1975 .

Biskamp, D., and R. Chodura, Asymptotic behavior of the two-stream instability, Phys. Fluids, 16, 888, 1973.

Block. L. P., Coulping Between the Outer Magnetosphere and the High-Latitude Ionosphere, Space Science Rev., 7, 198, 1967.

Block, L. P., Fotential double layers in the ionosphere, Cosmic Electrodynamics, $3,349,1972$

Block, L. P., Double layers, in B. Hultquist and I. Stenflo (eds.), Physjes of the Hot Plasma in the Magzetosphere, Plenum Press, New York, pp. $229-249,1975$.

Block, L.P., and C.-G. Fälthammar, Effects of Field-Aligned Currents on the Structure of the Ionosphere, J. Geophys. Res., 13, 4807,1968 . 
Block, L.P., and C.-G. Fälthammar, Mechanisms that may support magnetic-field-aligned electric fields in the magnetosphere, Ann. Geophys: 32, 1926, 1976.

Bosqued, J. M., G. Carđona, and̉ H. Rème, Auroral Electron Fluxes Parallel to the Geomagnetic Field Lines, J. Geophys. Res., 79, 98, 1974.

Boström, R., A Model of the Auroral Electrojects, I. Geophys. Res., 69, $4983,1964$.

Buneman, 0., Dissipation of Currents in Ionized Media, Phys. Rev., 115, 503,1959 .

Burch, J. L., S. A. Fields, W. B. Hanson, R. A. Heelis, and R. A. Hoffman, Characteristics of Auroral Electron Acceleration Regions Observed by Atmosphere Explorer C, J. Geophys. Res., 81, 2223, 1975.

Cauffman, D. P., and D. A. Gurnett, Satellite Measurements of High Latitude Convection Electric Fields, Space Science Rev., 13, 369, 1972

Chamberlain, J. W., Physics of the Aurora and Airglow, Academic Press, New York and London, P. 124, 1961.

Chappe11, C. R., Recent Satellite Measurements of the Morphology and Dynamics of the P1asmasphere, Rev. of Geophys, and Space Phys., 10, 951, 1972.

Choy, L. W., R. L. Arnoldy, W. Potter, P. Kintner, and I. J. Cahil1, Jr, Field-Aligned Particle Currents near an Auroral Arc, J. Geophys. Res., 76, 8279, 1971 .

Cloutier, B. A., B. R. Sandel, H. R. Anderson, P. M. Pazich, and R. J. Spiger, Measurements of Auroral Birkeland Currents and Energetic Particle Fluxes, J. Geophys. Res., 78, 640, 1973.

Cummings, W. D., R. J. O'Sullivan, and P. J. Coleman, Jr, Standing Alfvển Waves in the Magnetosphere, J. Geophys. Res., 74, 778, 1969.

Duncke1, N., B. Ficklin, L. Rorden, and R. A. Helliwe11, Low-Frequency Noise Observed in the Distant Magretosphere with Ogo-I, J. Goephys. Res., 75, 1854,1970 .

Evans, D. S., Precipitating Electron Fluxes Formed by a Magnetic FieldAligned PotentiaI Difference, J. Geophys. Res., 79, 2853, 1974.

Frank, I. A., Relationship of the Plasma Sheet, Ring Current, Trapping Boundary, and Plasmasphere near the Magnetic Equator and Local Midnight, I. Geophys. Res., 76, 2265, 1971 .

Frank, L. A., and K. L. Anderson, Observations of Charged Particle Precipitation into the Auroral Zone, J. Geophys. Res., 76, 3612, 1971.

Frank, I. A., and D. A. Gurnett, Distributions of Plasmas and Electric Fields over the Auroral Zones and Polar Caps, J. Geophys. Res.s 76, $6829,1971$. 
Gary, S. P., D. Montgomexy, and D. W. Swift, Particle Acceleration by Electrostatic Waves with Spatially Varying Phase Velocities, J. Geophys. Res., 73, 7524, 1968 .

Gurnett, D. A., The Earth as a Radio Source: Terrestrial Kilometric Radiation, I. Geophys. Res., 79, 4227, 1974.

Gurnett, D. A., and S.-I. Akasofu, Electric and Magnetic Field Observations During a Substorm on February 24, 1970, I. Geophys. Res. , 79, 3197, 1974.

Gurnett, D. A., and L. A. Frank, Observed Relationship between Electric Fields and Auroral Particle Precipitation, J. Geophys. Res., 78, 145, 1973.

Haerendel, G., A Note on Shear Flows in the Magnetosphere, Trans. Am. Geoph. Union, 55, 1005, 1974.

Hagg, E. L., Electron Densities of 8-100 Electrons $\mathrm{cm}^{-3}$ Deduced from Alouette II High-Iatitude Ionograms, Canadian J. of Phys., 45, 27, 1966.

Hallinan, T. J., and T. N. Davis, Small-scale auroral arc distortions, Planet. Space Sci., 18, 1735, 1970.

Hanson, W. B., D. R. Zuccaro, C. R. Lippincott, and S. Sanatani, The retarding-potential analyzer on Atmospheric Explorex, Radio Sci., $\underline{8}$, $333,1973$.

Heikkila, W. J., Outline of a Magnetospheric Theory, J. Geophys. Res., 79, 2496, 1974.

Helliwel1, R. A., A Theory of Discrete VLF Emissions from the Magnetosphere, J. Geophys. Res., 72, 4773, 1967.

Heppner, J. P., Electric Field variations during substorms: Ogo-6 measurements, P1anet. Space Sci., 20, 1475, 1972.

Heppner, J. P., High latitude electric fields and the modulations related to interplanetary magnetic field parameters, Radio Sci., 8, 933, 1973.

Hess, W. N., M. G. Triche1, T. N. Davis, W. C. Beggs, G. E. Kraft, E. Stassinopoulos, and E. J. R. Maier, Artificial Aurora Experiment: Experiment and Principal Results, J. Geophys. Res., 76, 6067, 1971.

Hoffman, R. A., and D. S. Evans, Field-Aligned Electron Bursts at High Latitudes Observed by Ogo-4, J. Geophys. Res., 73, 6201, 1968.

Hultquist, B., On the production of a magnetic-field-aligned electric field by the interaction between the hot magnetospheric plasma and the cold ionosphere, Planet. Space Sci., 19, 749, 1971.

Jaggi, R. K, , and R. A. Wolf, Self-Consistent Calculation of the Motion of a Sheet of Ions in the Magnetosphere, J. Geophys. Res., 78, 2852, 1973.

Jeffries, R. A., W. H. Roach, E. W. Hones, Jr, E. M. Wescott, H. C. StenbaekNielsen, T. N. Davis, and J. D. Winningham, Two Barium Plasma Injections into the Northern Magnetospheric Cleft, Geophys. Res. Lett., 2, 285, 1975. 
Jones, T. W., and P. J. Kellogg, Plasma Waves Artificially Induced in the Ionosphere, J. Geophys. Res., 78, 2166, 1973.

Kan, J. R., Energization of Auroral Electrons by Electrostatic Shock Waves, J. Geophys. Res., 80, 2089, 1975.

Kennel, C. F., and H. B. Petschek, Linit on Stably Trapped Particle Fluxes, J. Geophys. Res., 71, 1, 1966.

Kindel, J. M., and C. F. Kennel, Topside Current Instabilities, J. Geophys. Res., 76, 3055, 1971.

Knight, S., Farallel electric fields, Planet. Space Sci., 21, 741, 1973.

Kurth, W. S., M. M. Baumback, and D. A. Gurnett, Direction-Finding Measurements of Auroral Kilometric Radiation, J. Geophys. Res., 80, 2764, 1975.

Laval, G., and R. Pellat, Particle Acceleration by Electrostatic Waves Propagating in an Inhomogeneous Plasma, J. Geophys. Res., 75, 3255, 1970.

Lemaixe, J., and M. Scherer, Plasma sheet particle precipitation: a kinetic model, P1anet. Space Sci., 21, 281, 1973a.

Lemaire, $J$, , and M. Scherer, Kinetic Models of the Solar and Polar Winds, Rev. of Geophys. and Space Phys., 11, 427, 1973b.

Lemaire, J., and M. Scherer, Ionosphere-plaswasheet field-aligned currents and parallel electric fields, PIanet. Space Sci., 22, 1485, 1974.

Lennartsson, W., On high-latitude convection field inhomogeneities, Birkeland currents and inverted " $\nabla$ " precipitation events, techn. rep. TRITA-EPP-73-11, Department of Plasma Physics, Royal Inst, of Technology, S-100 44 Stockholm, 1973a.

Lennartsson, W., Ionospheric electrie field and current distribution associated with high altitude electric field inhomogeneities, planet Space Sci., 21. 2089,19736 .

McIlwain, C. E., Auroral electron beams near the magnetic equator, in B. Hultqvist and I. Stenflo (eds.), Physics of the Hot Plasma in the Magnetosphere, Plenum Press, New York, pp. 91-112, 1975.

O'Brien, B. I., Considerations that the source of auroral energetic particles is not a parallel electrostatic field, planet Space Sci., 18, 1821, 1970.

$O^{\prime}$ Brien, B. J., and D. L. Reasoner, Measurements of Highly Collimated Short-Duration Bursts of Auroral Electrons and Comparison with Existing Auroral Models, J. Geophys. Res., 76, 8258, 1971.

Park, R. J. and P. A. Cloutier, Rocket-Based Measurement of Birkeland Currents Related to an Auroral Arc and Electrojet, J. Geophys. Res., 76, 7714, 1971.

Perkins, F. V., Plasma-Wave Instabilities in the Ionosphere Over the Aurora, J. Geophys. Res., 73, 6631, 1968.

Persson, H., Electric Field Along a Magnetic Line of Force in a Low-Density Plasma, Phys. Fluids, 6, 1756, 1963. 
Persson, H., Electric Field Parallel to the Magnetic Field in a Low-Density Plasma, Phys. Fluids, 9, 1090, 1966.

Potter, W. E., Rocket Measurements of Auroral Electric and Magnetic Fields, I. Geophys. Res., 75, 5415, 1970.

Reasoner, D. L., and C. R. Chappe11, Twin Payload Observations of Incident and Backscattered Auroral Electrons, J. Geophys. Res., 78, 2176, 1973.

Rosenberg, T. J., R. A. Helliwe11, and J. P. Katsufrakis, Electron Precipitation Associated with Discrete Very-Low-Frequency Emissions, J. Geophys. Res., 76, 8445, 1971.

Sharber, J. R., and W. J. Heikkila, Fermi Acceleration of Auroral Particles, J. Geophys. Res., 77, 3397, 1972.

Shelley, E. G., R. G. Johnson, and R. D. Sharp, Satellite Observations of Energetic Heavy Ions During a Geomagnetic Storm, J. Geophys. Res., 77, 6104,1972 .

Stix, T. H., The Theory of Plasma Waves, McGraw-Hill, New York, 1962.

Swift, D. W., A Mechanism for Energizing Electrons in the Magnetosphere, J. Geophys. Res., 70, 3061, 1965.

Swift, D. W., Particle Acceleration by Electrostatic Waves, J. Geophys. Res., $75,6324,1970$.

Swift, D. W., On the Formation of Auroral. Arcs and Acceleration of Auroral Electrons, J. Geohpys. Res., 80, 2096, 1975.

Vasyliunas, V. M., Low Energy Particle Fluxes in the Geomagnetic Tail, in The Polar Ionosphere and Magnetospheric Processes, ed. G. Sk1ovi, Gordon and Breach, New York, 1970.

Venkatarangan, P., J. R. Burrows, and I. B. McDiarmid, On the Angular Distributions of Electrons in "Inverted-V" Substructures, J. Geophys. Res., $80,66,1975$.

Wescott, E. M., J. D. Stolarik, and J. P. Heppner, Electric Fields in the Vicinity of Auroral Forns from Motions of Barium Vapor Releases, J. Geophys. Res., 74, 3469, 1969.

Wescott, E. M., H. C. Stenbaek-Nielsen, T. J. Hallinan, T. N. Davis, and H. M. Peek, The Skylab Barium Plasma Injection Experiments. 2. Bvidence for a Double Layer, J. Geophys. Res., 81, 4495, 1976.

Westerlund, L. H,, The Auroral Electron Energy Spectrum Extended to $45 \mathrm{eV}$, J. Geophys. Res., 74, 351, 1969.

Thalen, B. A., and I. B. McDiarmid, Observations of Magnetic Field-Aligned Aurora1-E1ectron Precipitation, J. Geophys. Res., 77, 191, 1972.

Whipple, Ir., E.C., Relation between parallel-electric fielas and observed perticle velocity distributions, Phys. Dept., Univ. of Calif., San Diego, La Jolla, Ca. 92093, preprint, 1976 
Zxuda, A. J., and J. G. Armstrong, The Diurnal Variation of the Region with Vector Magnetic Field Changes Associated with Field -Aligned Currents, I. Geophys. Res., 79, 2501, 1974 .

Zmuda, A. J., J. C. Armstrong, and F. T. Heuring, Characteristics of Transverse Magnetic Disturbances Observed at $1100 \mathrm{~km}$ in the Auroral Oval, J. Geophys. Res., 75, 4757, 1970. 


\section{Figure Captions}

Figure 1.(a) The schematic electric field lines and equipotentials created at a resistant portion of an otherwise good conductor when a current is flowing. The surrounding medium is vacuum.

(b) The formal analogue when the resistor is a subregion of low conductivity within a magnetized plasma.

Figure 2. The electric field and current distributions at different altitudes associated with a given distribution of $E_{2}=E_{x}$ at altitude $z_{b}$, where $z_{b}$ is assumed to be in the topside ionosphere or low magnetosphere. The magnetic field is vertical and downward (antiparaliel to the z-axis).

Figure 3. The analogue of Figure 2 when $E_{l}$ at $z_{b}=1500 \mathrm{~km}$ has a smooth distribution and the parallel resistivity (averaged over altitude) above $z_{b}-h=1000 \mathrm{~km}$ is defined by the bottom curve.

Figure 4.

The various plasma regions expected when a large $(\Delta V) \|$ is being supported by magnetic mirroring of the charge carriers $e_{1}$, which are hot electrons. The high-altitude region 1 is devoid of cold particles and $E_{\|} \neq 0$ there, whereas the low-altitude region 3 has mainly cold particles and $E_{\|}=0$. Region 2 is a transition region where strong spacecharge exfects are likely to occur. The $E_{\|}$-field accelerates the electrons $e_{1}$, and precipitates some of them, while retarding hot protons $P_{1}$. At the same time $E_{\mid}$accelerates ionospheric protons $\mathrm{P}_{3}$ and reflects backscattered and secondary electrons $e_{3}$. The electrons $e_{2}$ are trapped by the $E_{\mid}$-field and the magnetic minror below.

Figure 5. A possible configuration of a magnetosphere-ionosphere current system (projected onto the dawn-dusk plane). The current paths $B-C, D-A$ and $A^{-}-D^{-}$as well as the dotted path are parallel to the magnetic field, while the remaining current paths are in the transverse direction. The dashed current path $A-W-A^{\prime}$ is within the solar wind. 
Figure $6 \quad$ A sketch of a case where the downward currents flow immediately outside of the upward current sheet (precipitating electrons). Only the upwand current is assumed associated with a small oll. The low-altitude $E_{x}$ is "shorted out" within the precipitation structure, as a result of the enhanced ionization. 


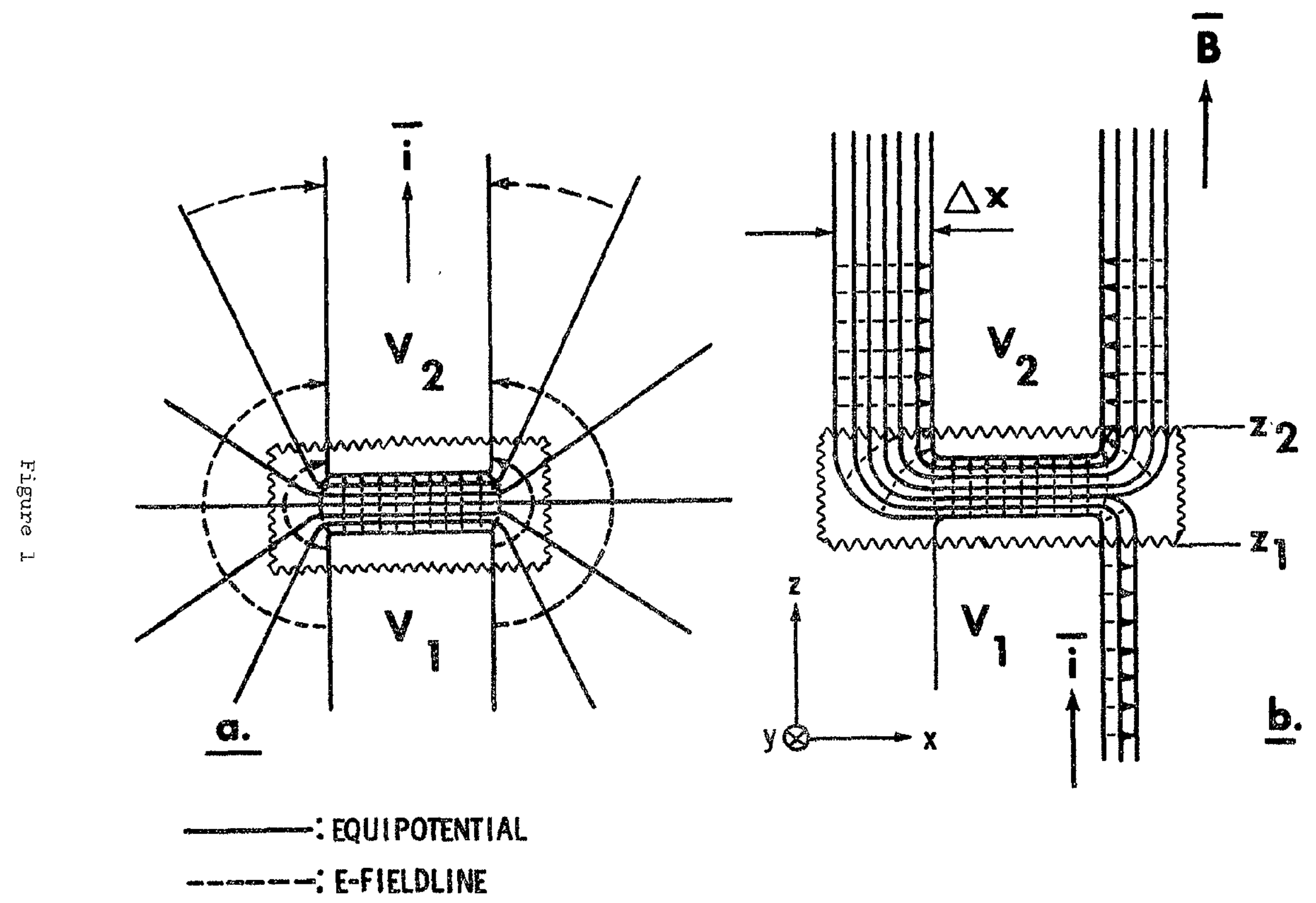



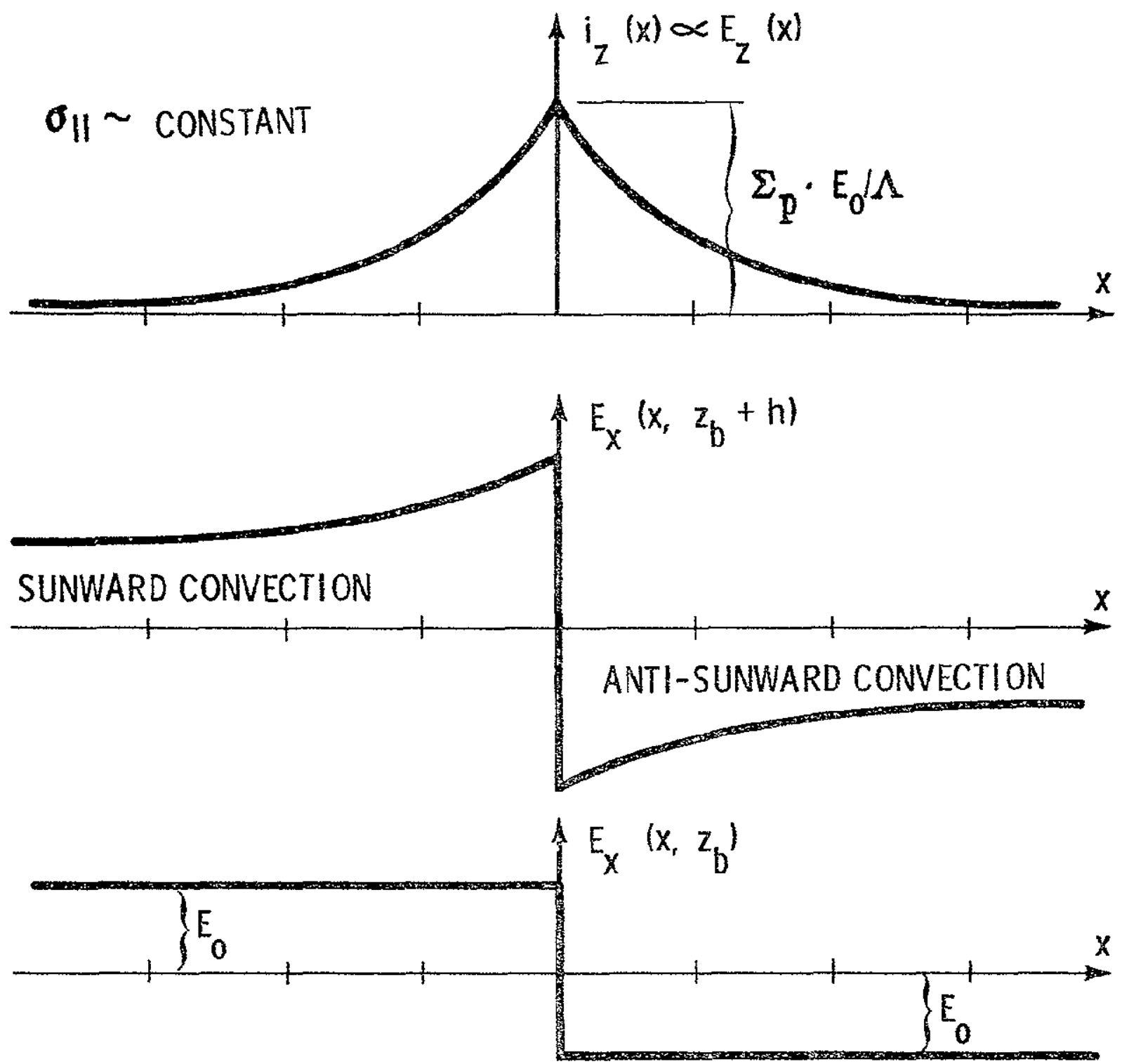

$$
\Lambda=\sqrt{\frac{h \cdot \Sigma \mathrm{p}}{\sigma_{11}}}
$$

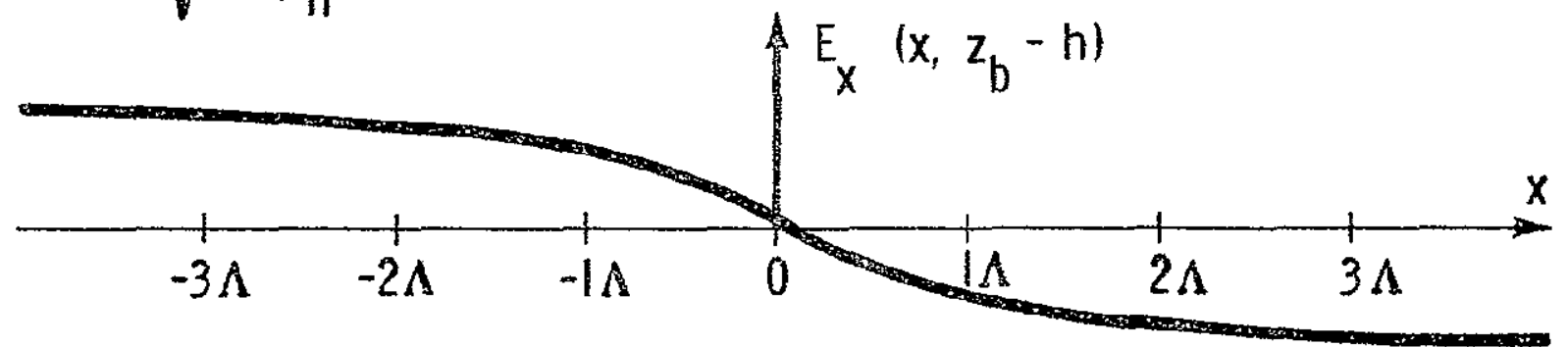

$\Sigma_{\mathrm{p} \sim \text { CONSTANT }}$ 


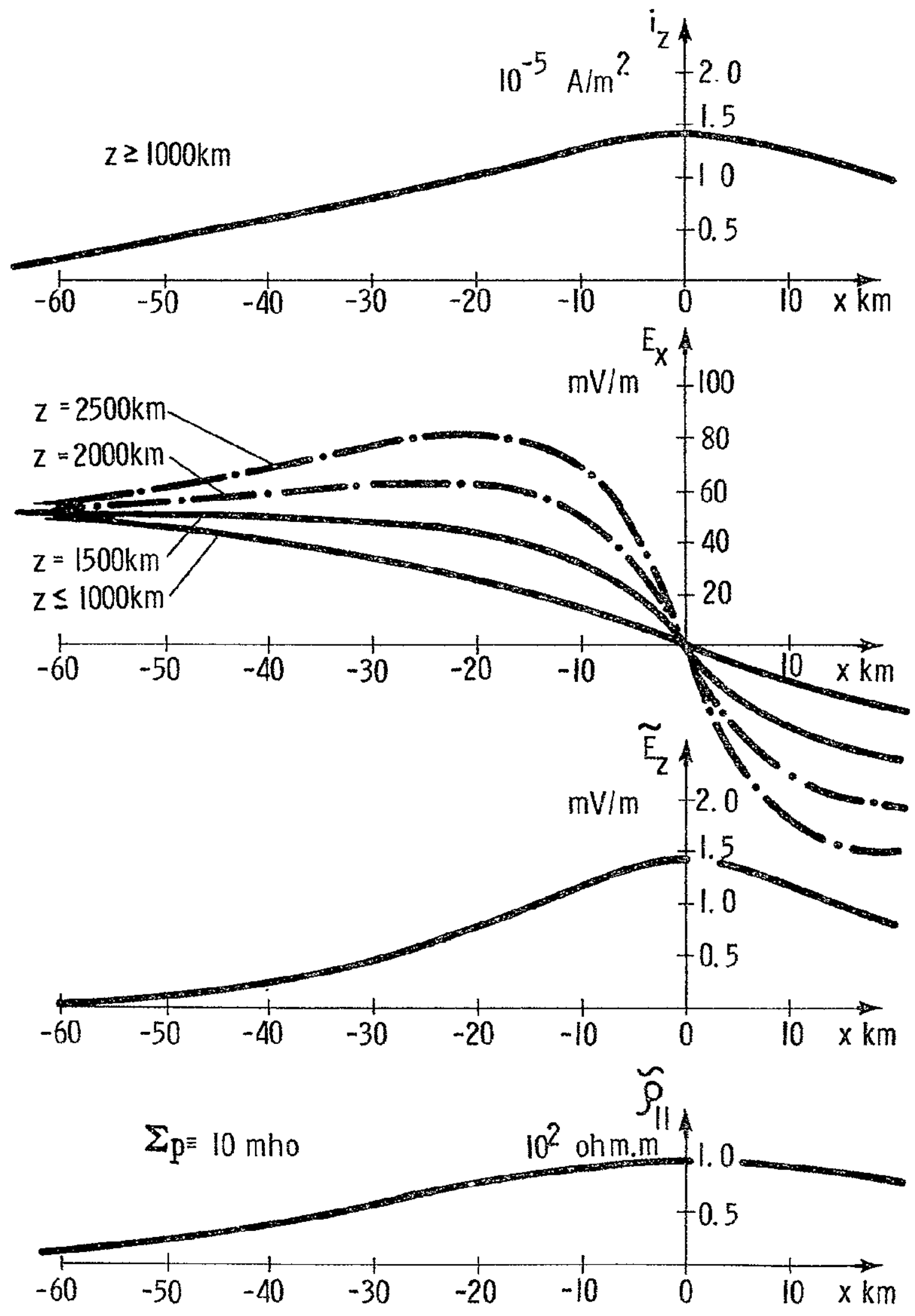

Figure 3 


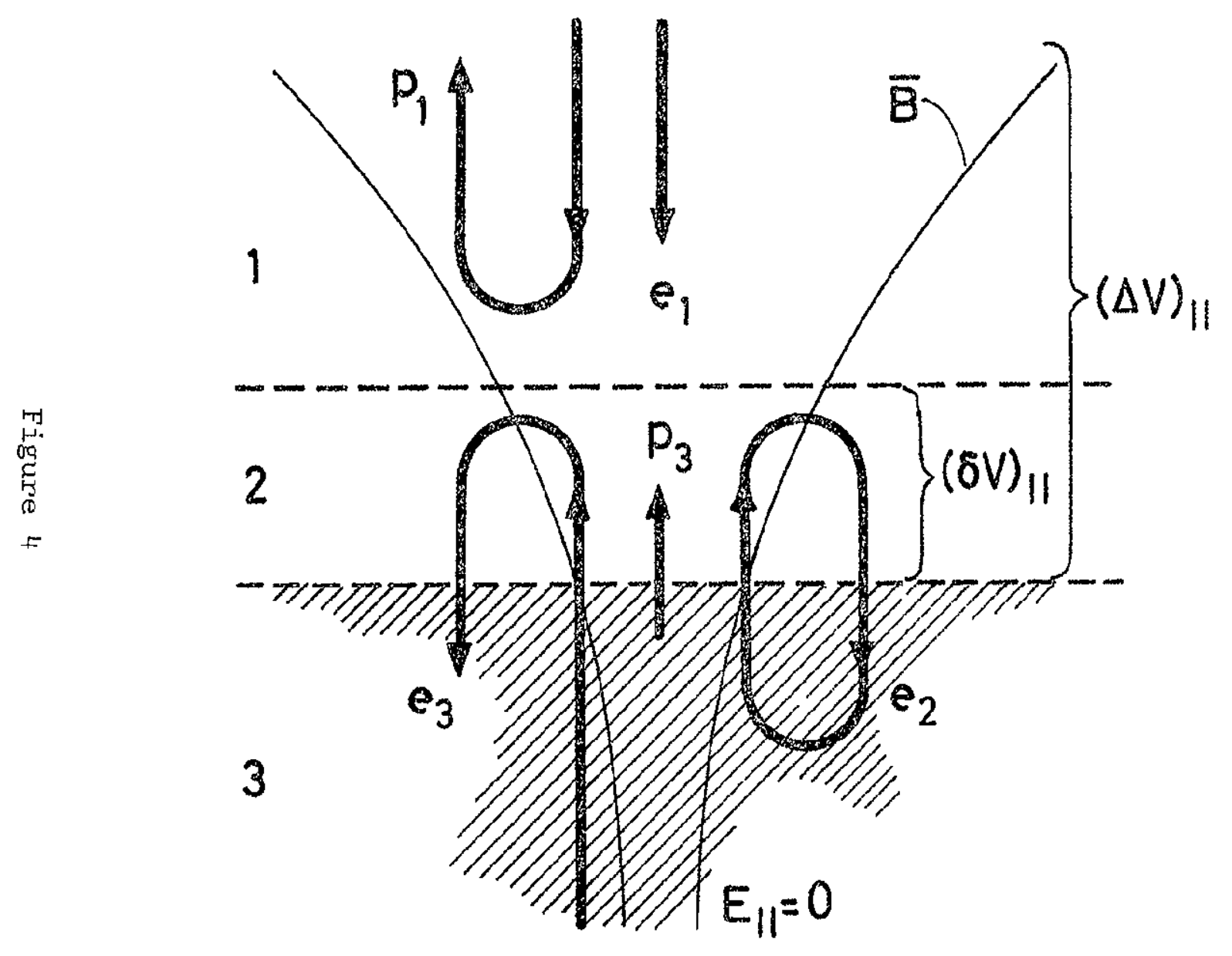




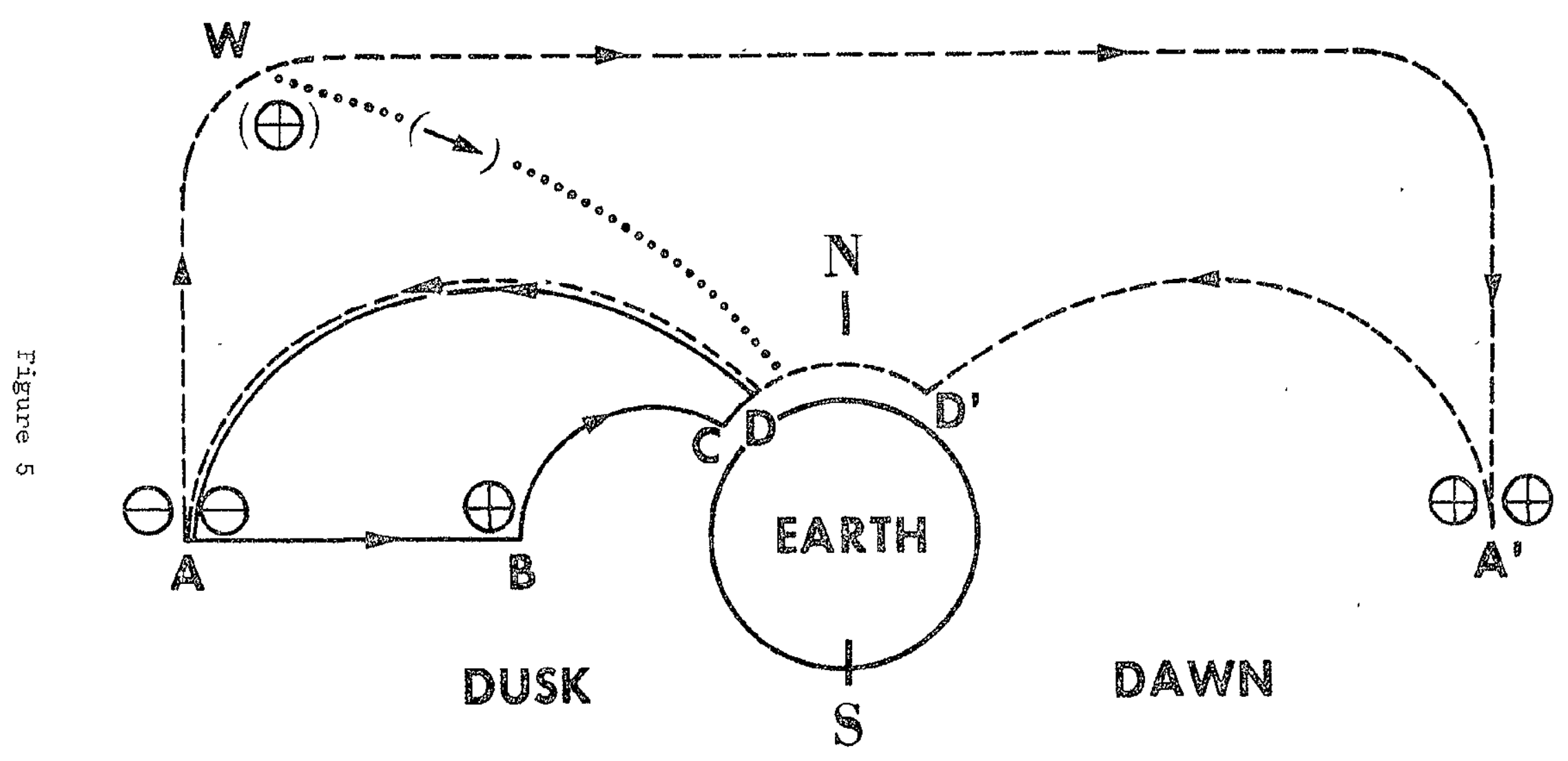




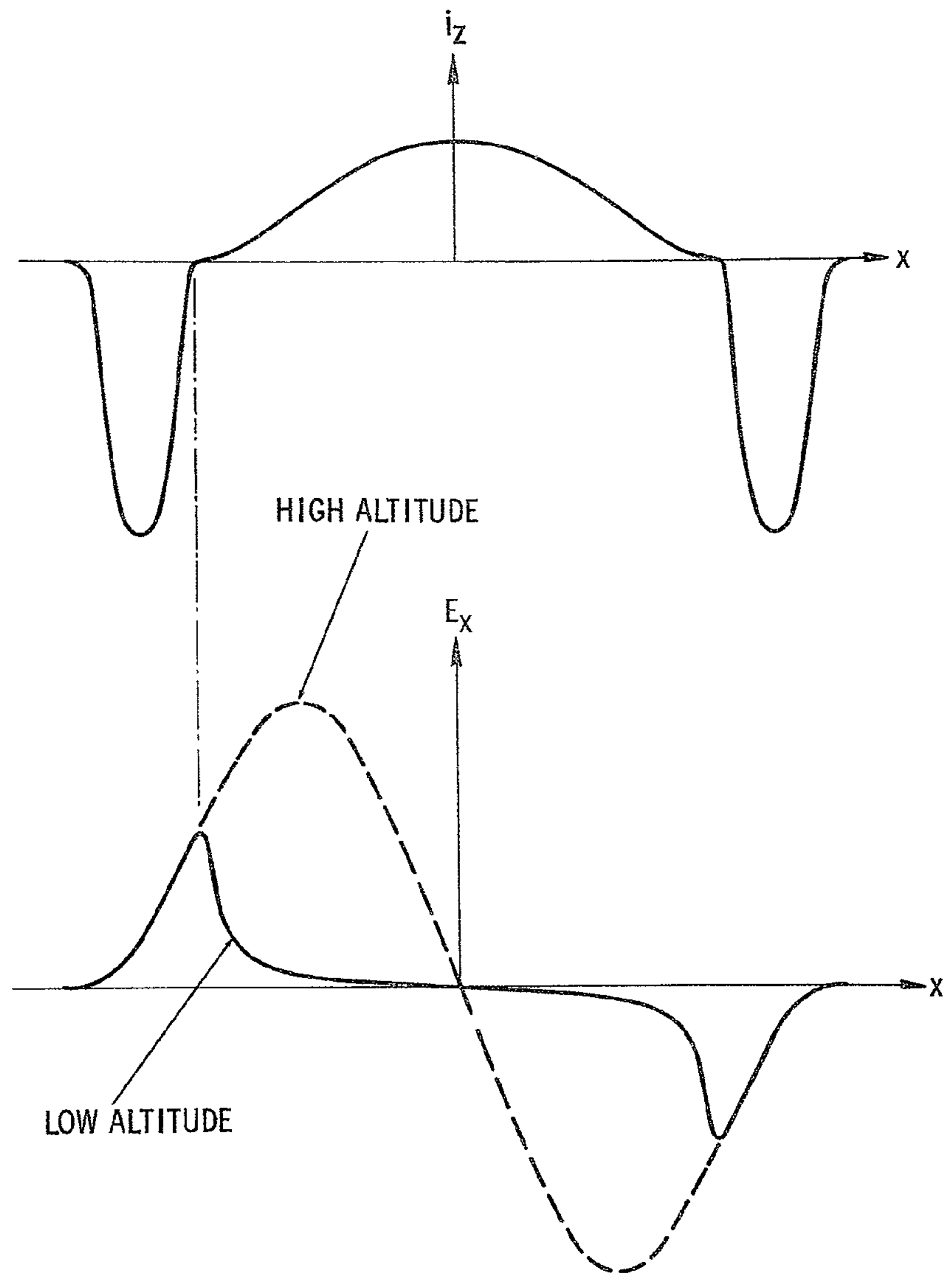

Figune 6 
TRITA-EPP-77-11

Royal Institute of Technology, Department of Plasma Physics, Stockholm 70, Sweden

ON THE ROLE OF MAGNETIC MIRRORING IN THE AURORAL PHENOMENA

WaIter Lennartsson

December 1976, 63 p. incl. ill., in English

On the basis of field and panticle observations, it is suggested that a bright auroral display is a part of a magnetosphere-ionosphere current system which is fed by a charge-separation process in the outer magnetosphere (or the solar wind). The upward magnetic-field-aligned current is flowing out of the display, carried mainly by downflowing electrons from the hot-particle populations in the outer magnetosphere (the ambient cold electrons being depleted at high altitudes). As a result of the magnetic mirroring of these downflowing current carriers, a large potential drop is set up along the magnetic field, increasing both the number flux and the kinetic energy of precipitating electrons. It is found that this simple basic model, when combined with wave-particle interactions, may be able to explain a highly diversified selection of auronal particle obsenvations. It may thus be possible to explain both "inverted- $V$ " events and auroral rays" in terms of a static parallel electric field, and the electric field may be compatible with a strongly variable pitchangle distribution of the precipitating electrons, including distributions peaked at $90^{\circ}$ as well as $0^{\circ}$. This model may also provide a simple explanation of the simultaneous precipitation of electrons and collimated positive ions.

Key words auroras, magnetosphere, electric fields, Birkeland currents, magnetic mirroring, anomalous nesistivity, double 